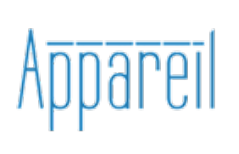

Appareil

12 | 2013

Walter Benjamin. Politiques de l'image

\title{
Mort pour être photographié
}

Le fonctionnement politique des photographies pendant les purges staliniennes

Denis Skopin

\section{(2) OpenEdition}

Journals

Édition électronique

URL : http://journals.openedition.org/appareil/1958

DOI : 10.4000/appareil.1958

ISSN : 2101-0714

Éditeur

MSH Paris Nord

Référence électronique

Denis Skopin, « Mort pour être photographié », Appareil [En ligne], 12 | 2013, mis en ligne le 16 décembre 2013, consulté le 30 juillet 2020. URL : http://journals.openedition.org/appareil/1958 ; DOI : https://doi.org/10.4000/appareil.1958

Ce document a été généré automatiquement le 30 juillet 2020.

\section{(c) $(1)(9)$}

Appareil est mis à disposition selon les termes de la Licence Creative Commons Attribution - Pas d'Utilisation Commerciale - Pas de Modification 4.0 International. 


\section{Mort pour être photographié}

Le fonctionnement politique des photographies pendant les purges

staliniennes

Denis Skopin

1 Ce texte a pour objectif d'analyser un phénomène qui se situe au croisement de deux sphères, esthétique et politique: le phénomène de la destruction des visages des « ennemis du peuple » sur les photos de groupe dans l'URSS des années trente. Lors des purges staliniennes ${ }^{1}$, certains visages furent noircis à l'encre, rayés, grattés ou découpés avec des ciseaux. Le plus souvent, les photographies étaient biffées par les proches de la victime, les membres de sa famille, ses amis ou ses collègues.

2 Cette interrogation conjointe doit permettre d'analyser sous un nouvel angle la question du rapport entre l'image et la politique. Si la recherche soulève la question du rapport entre ces deux sphères, ce n'est pas pour parler, une nouvelle fois, du contenu politique de l'image, de son implication dans une stratégie politique. Ce n'est pas la compatibilité d'une image avec un message politique qui nous intéresse ici. La recherche procède a contrario, s'intéressant davantage à leur incompatibilité, prenant pour point de départ l'interdit de l'image, une intolérance à son égard. Cette intolérance, cette violence qui s'exerce contre les images semble témoigner le mieux de leur caractère intrinsèquement politique. Si un régime totalitaire interdit une image, c'est qu'elle détient un pouvoir politique qui est incompatible avec celui du régime. Quelle est la nature de ce pouvoir des images? Pourquoi, précisément, ces images ont-elles été condamnées ou, mieux encore, qu'y a-t-il dans ces images qui les rend insupportables?

Répondre à cette question serait répondre à deux questions à la fois. D'un côté, ce serait déjà apporter bien des éclaircissements sur la nature du régime politique qui sanctionne ces images, ici, sur la nature du régime stalinien et sa terreur. De l'autre côté, ce serait en dire beaucoup sur le pouvoir politique de la photographie, son essence et sa destination sociale. Notre analyse va donc se déployer selon deux axes, esthétique et politique. 


\section{Les formes de la suppression de l'« ennemi » des photographies}

4 Si la façon dont on faisait disparaître la représentation de l'« ennemi » était toujours différente, on peut néanmoins isoler quelques formes les plus fréquentes de ce qu'on peut nommer l'« exécution en images ».

5 Souvent, celui qui s'attaquait à une photographie en noircissait, avec de l'encre, des zones entières, comme on l'observe sur ce collage dans l'esprit d'un constructivisme tardif (1), ou sur une autre photo qui représente les "travailleurs d'honneur » d'un important chantier, autrement dit un groupe de hauts fonctionnaires, membres du conseil de tutelle de ce chantier (2). Le nom du disparu était également supprimé.

\section{Photographie 1}

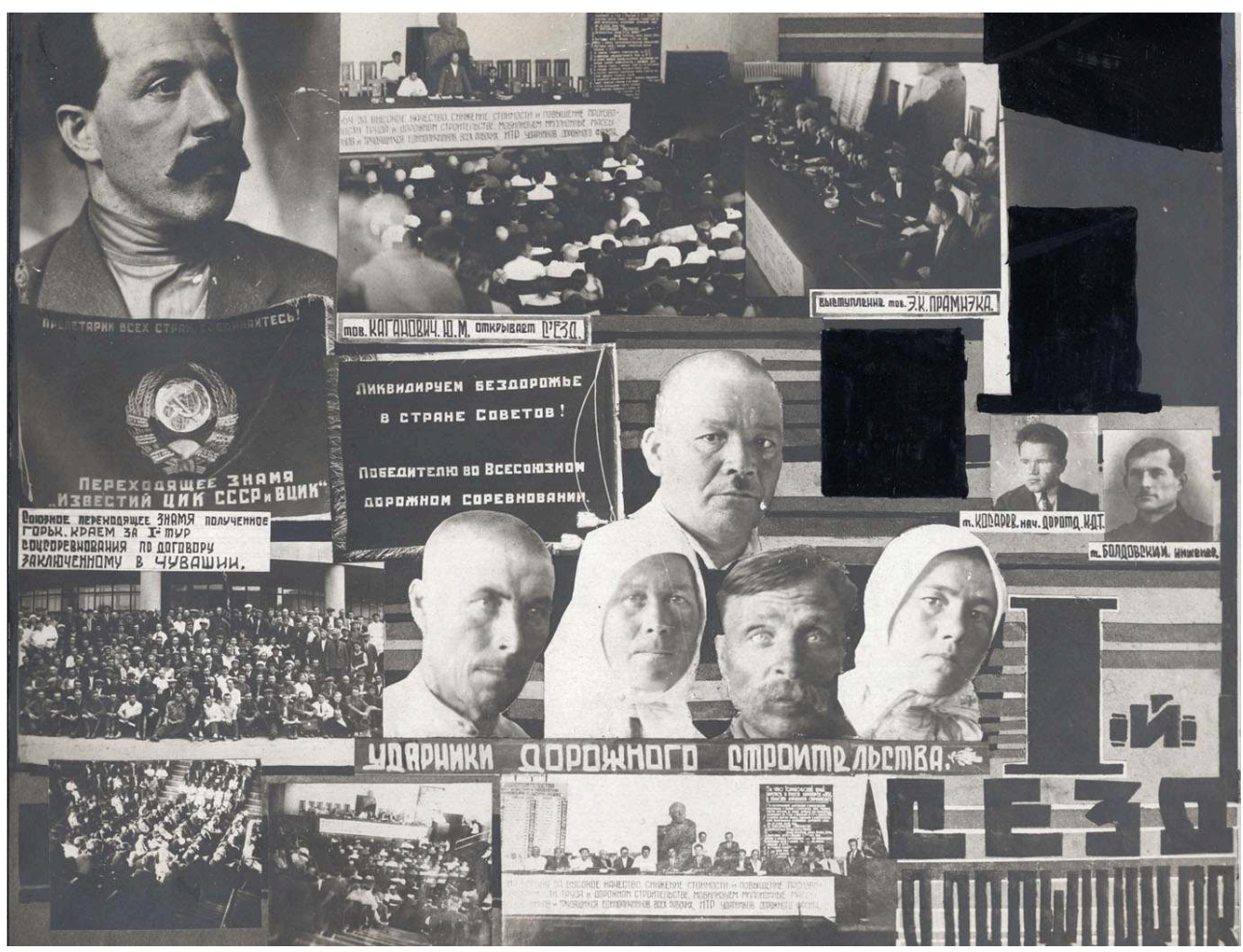

A. Ivanov, Allocutions de J. Kaganovitch et E. Pramnek au premier congrès des constructeurs de route. Portraits de travailleurs de choc, le 22 mai 1934. Archives audiovisuelles de Nijni-Novgorod // Album $n^{\circ} 12$, «Construction routière dans la Région de Gorky » (« Kraïdortrans »).

(c) Archives audiovisuelles de Nijni-Novgorod 


\section{Photographie 2}

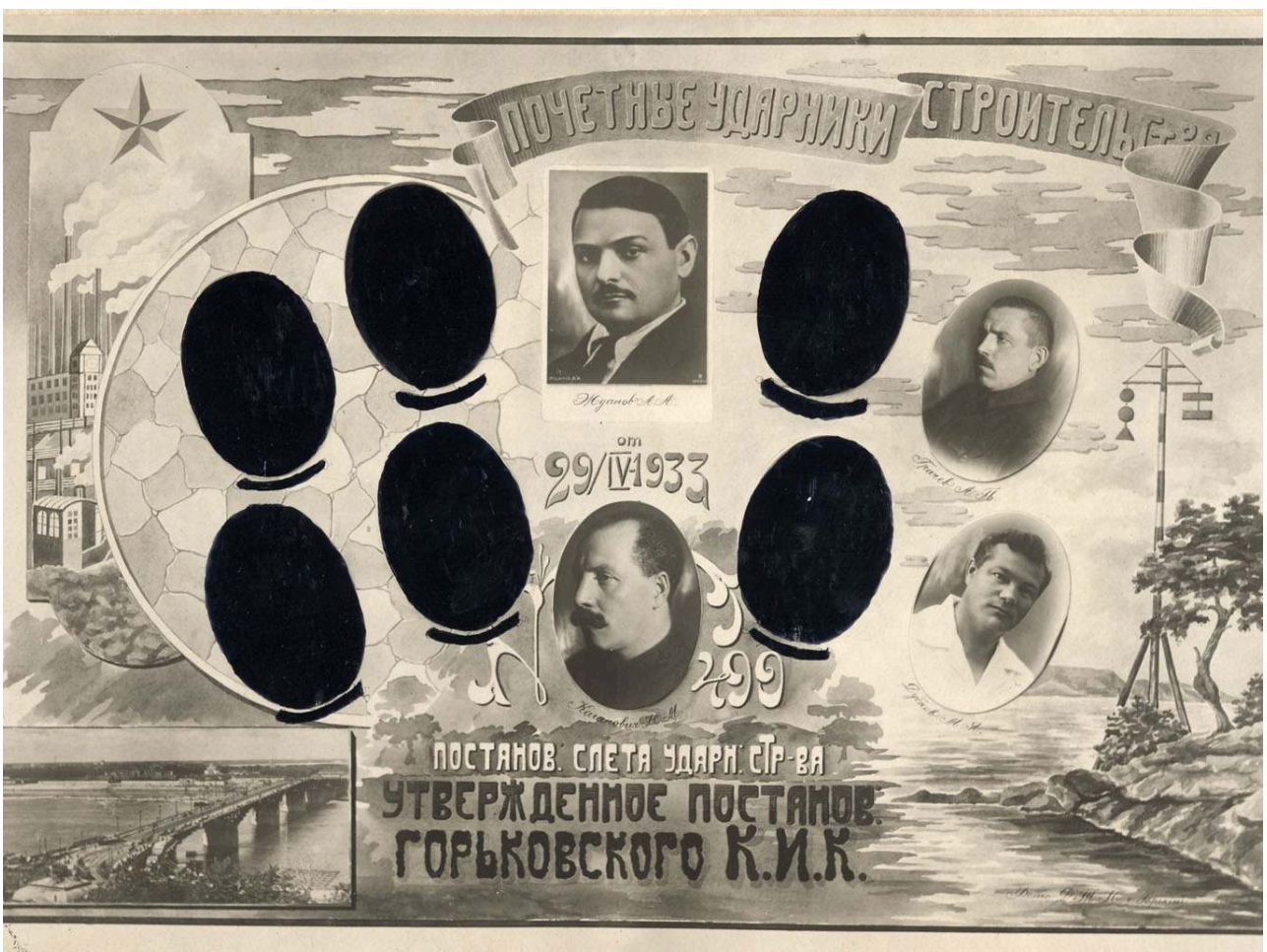

Anonyme, Le portrait de groupe des travailleurs d'honneur du chantier: A. Jdanov, J. Kaganovitch,

A. Gratchev, M. Doubnov. 29/IV1933. Archives audiovisuelles de Nijni-Novgorod / Album n 7, « Le pont Nikolas Pachomov traversant l'Oka à Gorky. 1930-1933 ».

(C) Photo DTK, Gorky. Archives audiovisuelles de Nijni-Novgorod

6 Ou bien l'attaque iconoclaste avait un caractère ponctuel, en se focalisant sur le visage de l'ennemi du peuple (3). 
Photographie 3

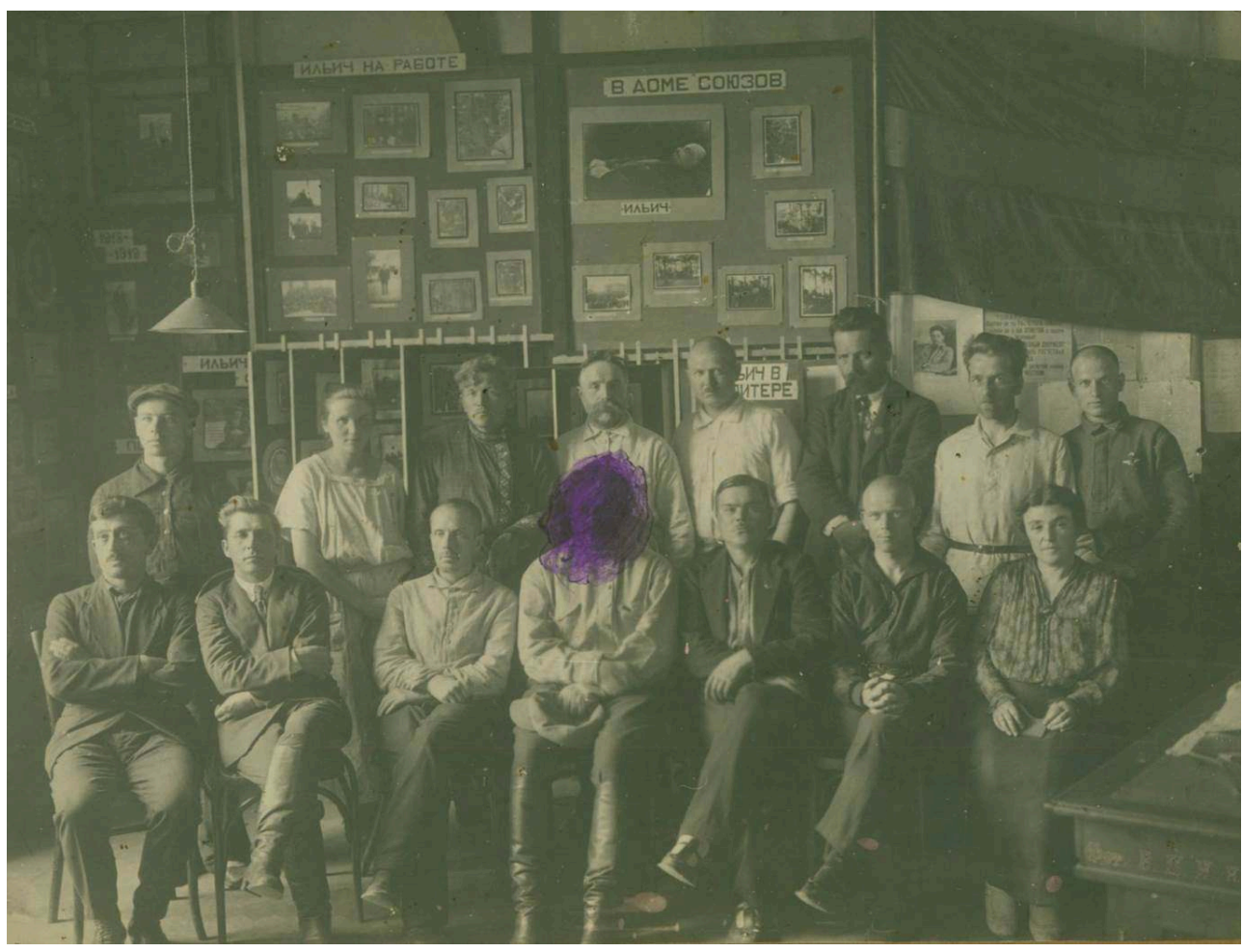

Photographie du dossier de Zoubarev, programme «ALGIR-2 »

(c) Archives de Mémorial, Moscou

7 Rayer en croix les visages des ennemis du peuple était, à l'époque stalinienne, l'autre forme - moins radicale que le caviardage - de leur exécution symbolique. C'est ce que nous voyons sur la photo suivante (4). 


\section{Photographie 4}

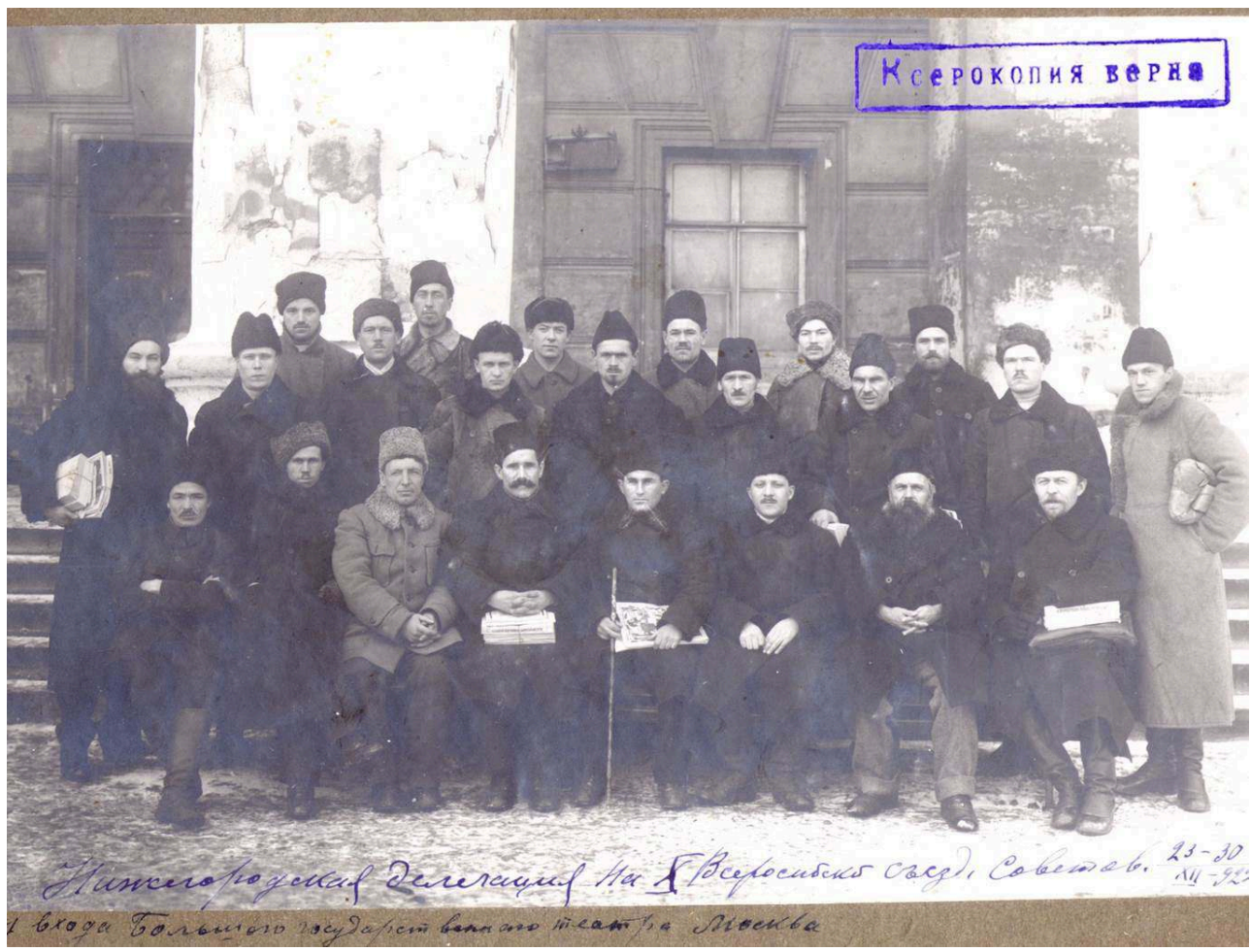

Anonyme, Les délégués de Nijni-Novgorod au Xe congrès des Conseils près de l'entrée du Théâtre Bolchoï: Machotine, I. Tchugurine, N. Uglanov, Kaganovitch, A. Taganov. Le 23 décembre 1930. Archives du Comité régional du Parti Communiste de Nijni-Novgorod / Fonds 7853, dossier 418, inventaire 1.

(c) Archives du Comité régional du Parti Communiste de Nijni-Novgorod

Plus tard, dans les années 1960 (au moment de la réhabilitation), les deux traits en croix sur les visages de deux personnes au centre ont été gommés. On l'observe très bien en agrandissant la partie centrale (5). 


\section{Photographie 5}

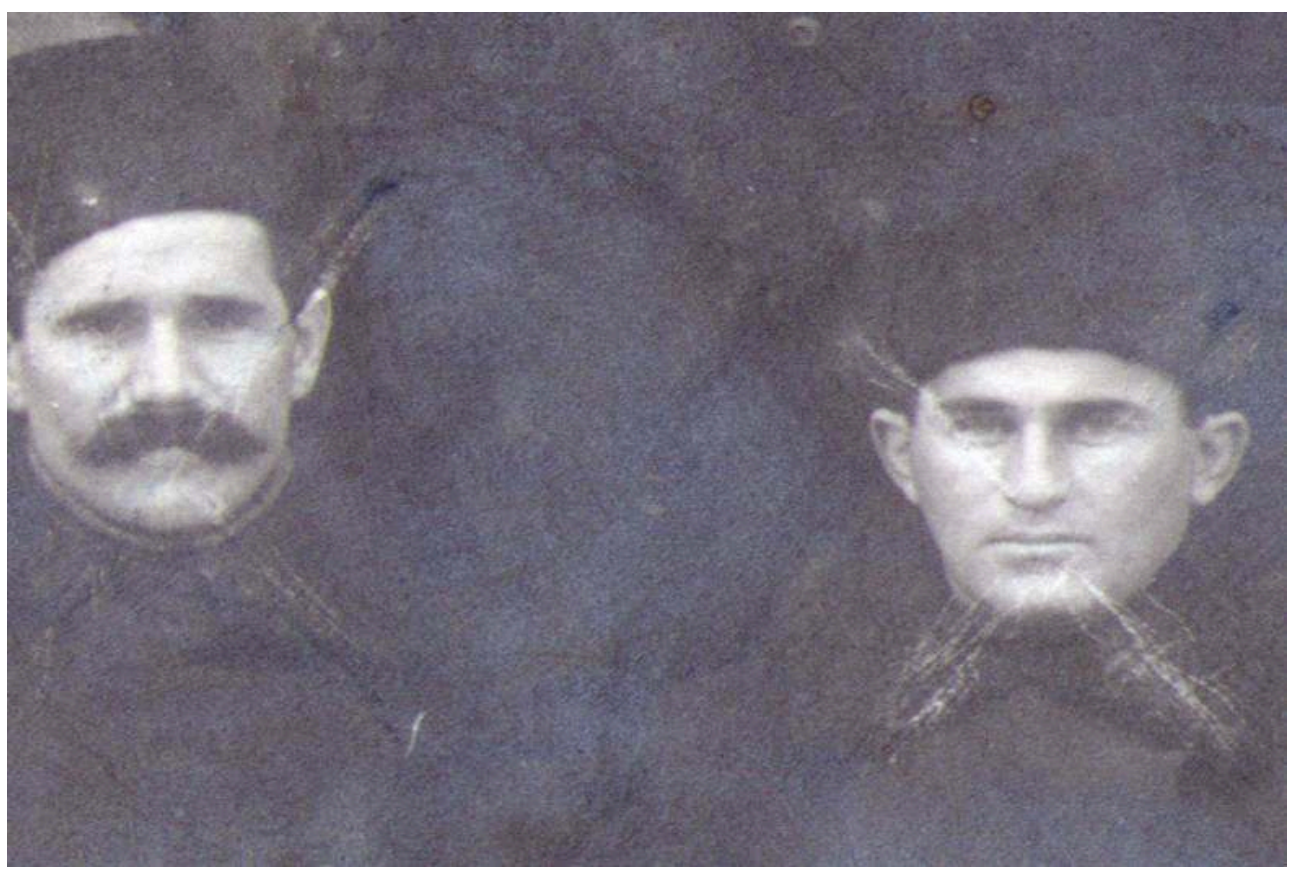

Anonyme, Les délégués de Nijni-Novgorod au Xe congrès des Conseils près de l'entrée du Théâtre Bolchoï: Machotine, I. Tchugurine, N. Uglanov, Kaganovitch, A. Taganov. Le 23 décembre 1930. Archives du Comité régional du Parti Communiste de Nijni-Novgorod / Fonds 7853, dossier 418, inventaire 1. (Fragment)

(c) Archives du Comité régional du Parti Communiste de Nijni-Novgorod

9 Parfois, la représentation de l'ennemi était découpée ou grattée (photographies 6 et 7). 
Photographie 6

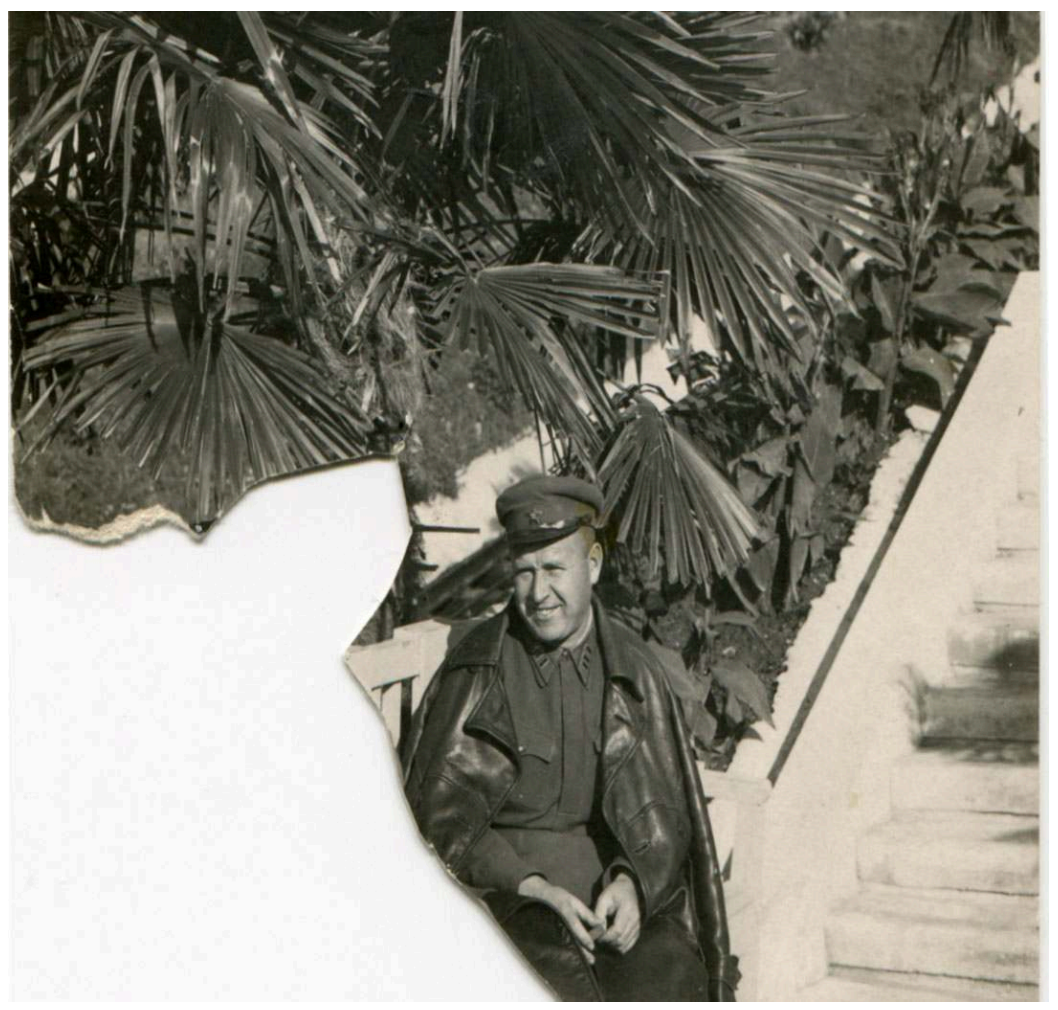

Photographie du dossier de Karmanov, Archives de Mémorial, Moscou

(c) Archives de Mémorial, Moscou 
Photographie 7

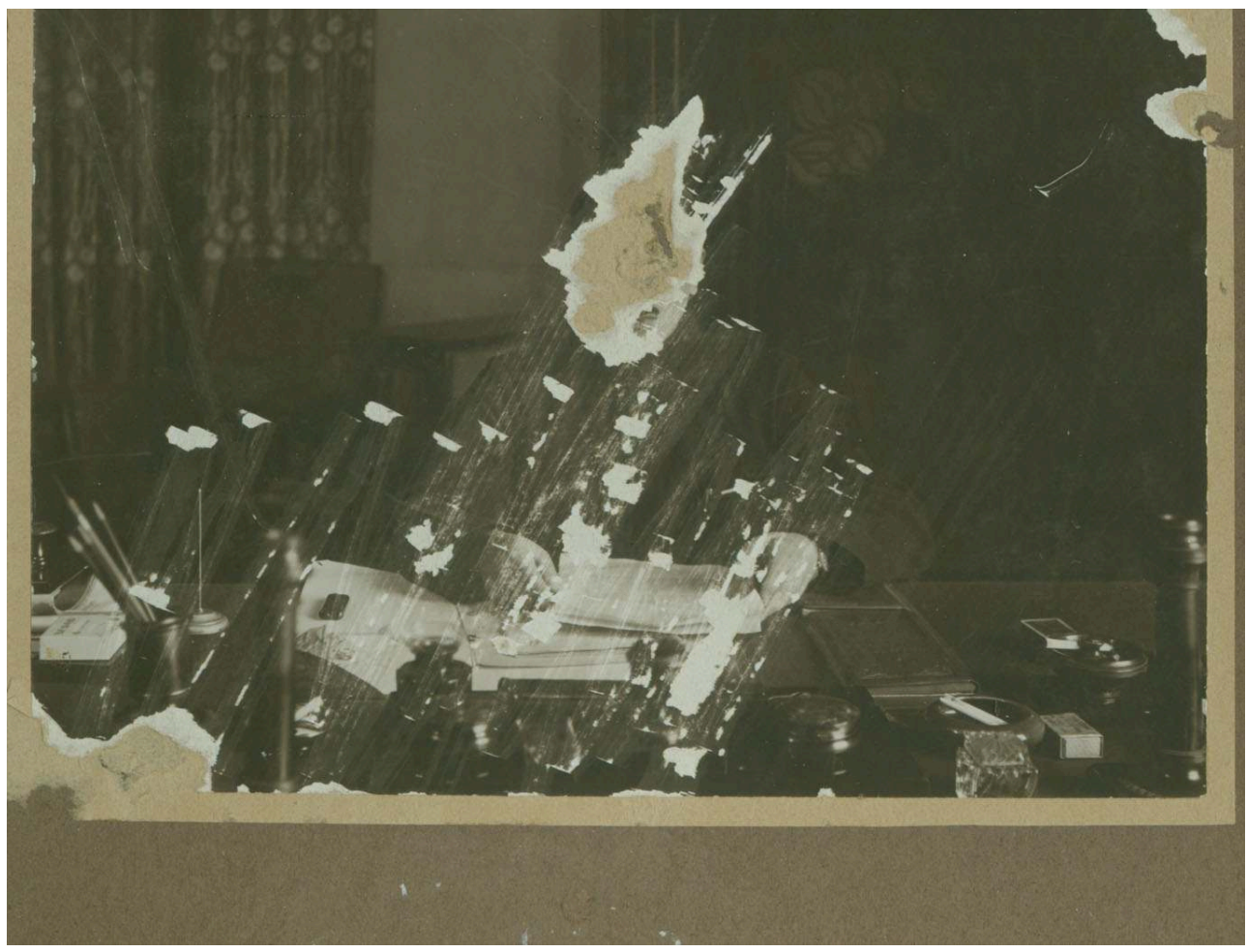

Photographie du dossier de Bérézine, programme «Photoscans », Archives de Mémorial (c) Archives de Mémorial

10 Puis, il y a des photographies, comme celle-ci (8), qui, après être noircies dans un premier temps, ont reçu un coup de ciseaux par la suite. On y voit encore à gauche et à droite des traces d'encre noire. 


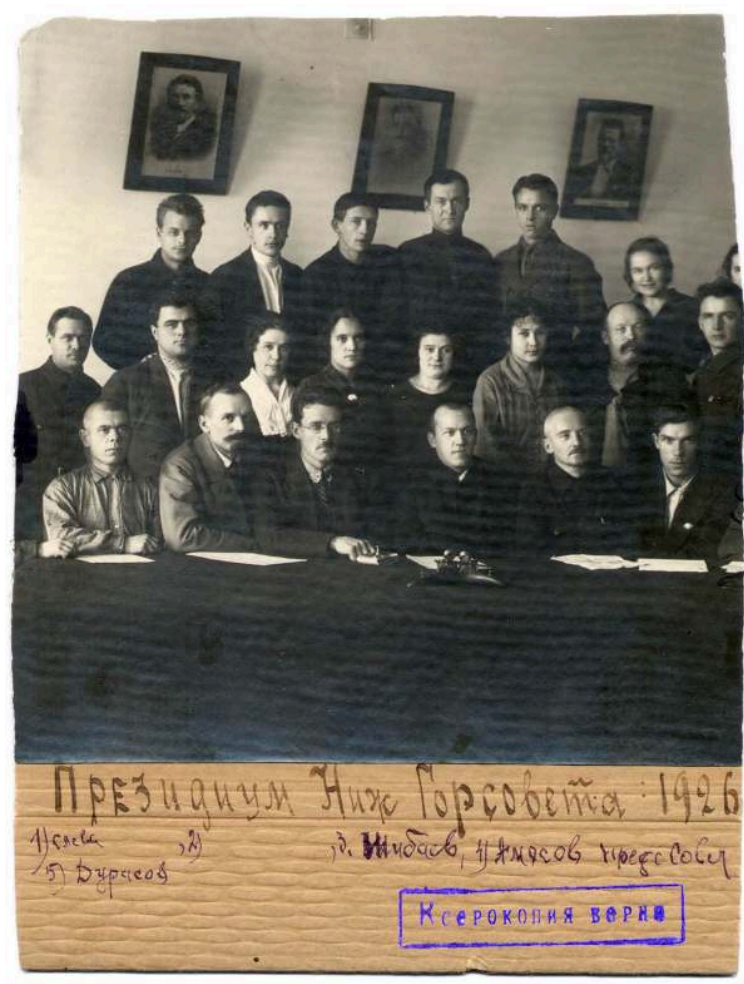

Anonyme. La présidence du Conseil municipal de Nijni-Novgorod: Durassov, Amossov, Chibaev. NijniNovgorod, 1926, Archives du Comité régional du Parti communiste de Nijni-Novgorod / Fonds 7853, dossier 387, inventaire 1.

(c) Comité régional du Parti communiste de Nijni-Novgorod

11 Puisque la différence des formes de l'élimination de l'ennemi a elle aussi de l'importance, je reviendrai là-dessus un peu plus tard.

\section{Les « doctrines de l'inimitié »}

12 Il paraît que la conjoncture historique et politique de la première moitié du $\mathrm{xx}^{\mathrm{e}}$ siècle ait ceci de particulier qu'elle serait difficile à décrire sans faire appel à la notion de "guerre ». L'historien Enzo Traverso semble avoir raison lorsqu'il décrit la période de 1914 à 1945 en Europe comme une période de guerre ininterrompue, en établissant une analogie entre ce laps de temps et la fameuse guerre de Trente Ans ${ }^{2}$. Quand il se met en place dans l'URSS stalinienne, le dispositif de la terreur puise sa légitimité dans la notion de guerre : la terreur se présente toujours comme une guerre que l'État mène contre un ennemi intérieur. La notion d'ennemi du peuple prend elle aussi tout son sens seulement dans le contexte d'une guerre permanente où se trouvent engagés l'État soviétique et ses citoyens.

Il faut donc prendre au sérieux les paroles de Carl Schmitt, le penseur qui a poussé le plus loin la réflexion sur la guerre et l'hostilité comme fondement de la vie politique, selon lequel, l'esprit de Hegel, éminent philosophe de la guerre, s'est déplacé de Berlin à Moscou, ce qui donne à Schmitt l'occasion de déplorer cette situation. Du même coup, Schmitt trace une généalogie de son propre discours et d'un discours "ennemi », laquelle est d'autant plus curieuse qu'elle remonte au-delà de l'opposition idéologique 
qui s'est dessinée au xxe siècle, pour faire voir leur parenté plus profonde. À l'en croire, l'État prussien préféra emprunter sa philosophie conservatrice à Friedrich Julius Stahl, alors que Hegel, selon l'expression de Schmitt, «rejoignait Lénine via Karl Marx et émigrait à $\mathrm{Moscou}^{3} »$. Schmitt estime que les théoriciens marxistes, avec leur notion d'« ennemi de classe », avancent plus loin que les autres dans la réflexion sur la guerre et l'inimitié. Pour confirmer cette thèse, Schmitt cite un nom, celui de Georg Lukács. C'est sans doute de Lukács ${ }^{4}$ que Schmitt tient son appréciation de l'activité politique de Lénine, à qui il rend hommage dans La théorie du partisan.

\section{La guerre et l'ennemi de classe selon Georg Lukács}

Schmitt a tout à fait raison de citer Lukács comme grand théoricien de l'hostilité. Malgré toutes ses divergences avec le stalinisme officiel et la critique de la part des partisans du marxisme vulgaire, Lukács a le mieux compris et formulé la dialectique de l'ami et de l'ennemi qui, au temps de Staline, imprègne le système des valeurs du citoyen soviétique, en définissant ses prises de position, ses sympathies et ses haines.

Le marxisme de Lukács, tel qu'on le trouve dans son célèbre essai La conscience de classe, est un marxisme qui se situe aussi loin que possible de tout académisme, de tout marxisme comme critique des inégalités sociales ou théorie de l'art, à l'instar du marxisme esthétisant et élitiste d'Adorno. La question qui se pose dans La conscience de classe, texte qui s'interroge sur les mécanismes de la lutte politique, est loin d'être abstraite. Il s'agit de la question pratique «comment vaincre?». Lukács part du présupposé qu'une classe, à condition qu'elle veuille arriver au pouvoir, doit être consciente de ses intérêts, car c'est cette conscience qui permet à un groupe de réunir ses efforts. Plus une classe est consciente de ses intérêts, plus elle a la chance de se consolider comme classe et de vaincre. Pour atteindre ses buts, une classe doit voir clairement les problèmes qui se posent devant elle, doit savoir distinguer ses intérêts et ses ennemis.

Mais il y a un facteur extrêmement important qui intervient ici : une classe ne prend conscience de ses intérêts que dans la guerre. Qu'une classe soit historiquement appelée à la domination ne signifie pas automatiquement qu'elle pourra réunir ses efforts et arriver au pouvoir. Il faut que la classe naissante s'engage dans une guerre, qui est l'autre condition de sa constitution définitive. La classe n'est pas une classe jusqu'à ce qu'il y ait de la communauté entre ceux qui doivent y appartenir. Mais cette communauté n'apparaît qu'en réaction contre un ennemi extérieur; la classe se constitue, « s'individue » au cours de la confrontation, face à un ennemi extérieur.

On voit que ce constat inverse l'opinion courante selon laquelle les acteurs du conflit préexistent au conflit. Selon Lukács, la guerre ne résulte pas de la confrontation des classes déjà constituées, au contraire, l'identité des intérêts de classe se dessine au moment de la confrontation. C'est ainsi que la violence qui a accompagné l'accumulation primitive du capital a créé les conditions pour la constitution de la bourgeoisie en tant que classe. L'identité d'un groupe politique ne serait que son reflet que lui retourne le miroir du groupe adverse: la conscience de classe est nécessairement changeante, car elle est " toujours une forme d'emprunt ${ }^{5}$. " L'identité d'un groupe se définit et se redéfinit en fonction de l'ennemi qui s'y oppose. 
Pour Lukács, la violence ne serait pas seulement un effet, ne serait pas, pour ainsi dire, un «mal inévitable » qui accompagnerait la lutte des classes. On découvre l'hostilité à l'origine des classes elles-mêmes, ce qui revient à dire à l'origine de la société. La guerre serait à l'origine de la vie sociale, elle serait son fondement, sa condition. Elle fonde le politique. La société bourgeoise désire effacer le fait de la lutte des classes de la conscience sociale, refouler le conflit qui déchire la société, l'enfouir sous les fauxsemblants de la paix. Pour Lukács, au contraire, il s'agit de faire voir la véritable origine de la société. De ce point de vue, la paix ne serait qu'une apparence, une fiction, une illusion. Par contre, la recherche de l'ennemi, si tant est que la vie sociale se fonde sur l'hostilité, serait une force motrice de toute société, y compris la société sans classes qu'est la société communiste. C'est pourquoi, après avoir triomphé sur ses ennemis de classe, le prolétariat devra rechercher un nouvel ennemi, cette fois en son propre sein ${ }^{6}$.

On voit jusqu'à quel point ce raisonnement de Lukács fait écho aux thèses de son lecteur, son adversaire politique et son ennemi de classe Carl Schmitt. Les différends idéologiques étant écartés, on retrouve au fond des deux théories une seule et même logique. Toutes les deux renversent la perspective traditionnelle : la guerre est déclarée être non seulement une façon de faire de la politique (point de vue de Clausewitz qui la considérait comme la prolongation de la politique par des moyens qui ne sont pas par eux-mêmes politiques), mais la condition de toute politique et même l'essence de toute politique. Indubitablement, Schmitt se montre plus radical et conséquent que son adversaire, l'auteur de la Conscience de classe. L'hostilité apparaît chez lui dépouillée de tout ce qui la cachait encore chez Lukács, où elle voisinait paradoxalement avec l'idéal marxiste d'une fraternité universelle.

20 Mais avant d'être un affrontement armé, la guerre est une optique déformante, une façon de percevoir où l'hostilité s'impose comme antérieure à l'amitié; dans cette optique, la paix apparaît comme improbable, étant tout au plus un armistice qui vient entrecouper l'état de guerre. Comme le remarque Levinas, l'état de guerre n'est pas une simple épreuve pour la morale. La « lucidité » qui met la guerre au cœur du politique a pour effet la suspension, ou bien la révocation de la morale qui apparait comme dérisoire. Cette vision qui se veut réaliste anticipe la morale, la présentant comme fruit d'une utopie idéaliste. La théorie de l'inimitié dénonce la paix comme illusion au profit de l'immédiateté d'un conflit originaire. Pourtant, ce rapport prétendument immédiat se révèle déjà médiatisé par une totalité, une finalité extérieure. Le rapport à l'autre dont parle Schmitt n'est jamais a priori, n'est jamais un rapport « tout court ». Il est déjà contaminé de totalité.

21 Certes, l'analyse de la dimension biopolitique de la doctrine schmittienne que propose Agamben est très importante. Agamben montre comment se creuse un vide juridique autour du homo sacer, un vide qui finalement rendra possible son extermination. Pourtant, on ne peut pas mésestimer l'importance de l'analyse des conséquences éthiques de cette doctrine, de l'analyse portant sur la suspension des impératifs moraux à l'égard de l'« ennemi ».

\section{La perte de l'ennemi et la « guerre moderne »}

Schmitt, on le sait, se prononce pour une guerre ouverte, déclarée, où l'adversaire est facile à localiser parce que les parties belligérantes sont séparées par une ligne de front. Pourtant, dans les conditions de la guerre moderne, la distinction entre ami et ennemi 
paraît problématique : la ligne de front tend à s'effacer et les antinomies classiques qui structuraient la guerre auparavant (régulier/irrégulier, légal/illégal, terre/ciel...) sont jugées inadéquates pour caractériser l'état de belligérance moderne: les différences entre elles s'effaceraient pour dessiner un nouveau profil de la guerre comme conflit latent et permanent.

Les théoriciens de la guerre moderne (le général Erich Ludendorff en Allemagne, le colonel Roger Trinquier en France) sont unanimes à mettre en relief l'importance qu'a le rétablissement de la ligne de front entre " amis » et « ennemis ». Pour vaincre, il est indispensable de situer l'adversaire. Une fois identifié, l'adversaire est facile à «neutraliser », car la guerre redevient ce qu'elle doit être. Comme le dit Trinquier, la neutralisation de l'adversaire n'est qu'un problème « technique ».

\section{L'identification de l'ennemi}

Dire que, dans les conditions de la guerre moderne, la ligne de front demeure invisible, impalpable, immatérielle, revient à postuler qu'elle est idéologique. C'est l'idéologie qui constitue la ligne de partage dans la guerre moderne. D'où le fait que les adversaires peuvent appartenir à la même nation, habiter le même village, être amis et voire membres de la même famille. Désormais, la ligne de front peut passer au sein d'une communauté d'amis ou d'une famille. L'ennemi dans la guerre moderne - et la société soviétique a toujours été, à toutes les périodes de son histoire, en état de guerre - est par excellence un ennemi intérieur.

Il s'ensuit que l'identification de l'adversaire cesse d'être une opération purement militaire pour devenir une opération idéologique. Le suspect doit prouver son innocence, sa loyauté en prenant une position politique claire, en se rangeant du «bon » côté du front politique.

C'est ici que surgit le recours aux photographies. Dès son apparition, la photographie a contribué à l'affirmation d'une nouvelle forme de la communauté «transindividuelle ».

\section{La transindividualité et sa nature symbolique selon Gilbert Simondon}

La notion qui semble le mieux caractériser la communauté qui s'instaure entre ceux qui co-apparaissent sur une photographie, c'est la notion de transindividualité qu'on trouve chez Gilbert Simondon. La conception simondonienne du milieu transindividuel a l'avantage de rompre avec les théories substantialistes de la subjectivité. Simondon met en question le caractère individuel de la spiritualité humaine, en écartant les doctrines substantialistes au profit d'une spiritualité relationnelle. Simondon, en quelque sorte, situe le propre de l'individu hors de lui, à la croisée de l'individuel et du collectif. La notion qui sert à Simondon à décrire les processus de subjectivation, c'est celle d'individuation. L'individuation est une concrétisation d'un objet ou d'un organisme au sein d'un système. D'une part, l'homme s'individue au sein d'un collectif, sans lequel l'individuation serait impossible; d'autre part, la collectivité évolue, "s'individue » de par chacun de ses membres. Pour Simondon, l'individuation a pour force motrice l'émotion que partagent les membres de la communauté. C'est plutôt 
l'émotion que la proximité intellectuelle qui constitue la clé de voûte du collectif, de la communauté transindividuelle.

Ainsi la communauté transindividuelle est-elle une sorte de réseau qui réunit les individus. Mais ce réseau n'est pas fait de ponts jetés d'une personne à l'autre, car il constitue les individus. Il ne s'agit en aucun cas d'un rapport « social », qui est toujours complémentaire et qui vient, pour ainsi dire, s'ajouter à l'individu de l'extérieur. Le rapport transindividuel est à distinguer du rapport social, interindividuel. C'est un rapport qu'on ne peut pas séparer de l'individu sans le détruire. Simondon fait la distinction entre symbole et signe : si le signe est complémentaire par rapport à l'objet, le symbole, lui, le constitue. S'il faut donner un exemple d'une communauté transindividuelle, ce sera une famille ou un groupe d'amis. Les rapports symboliques sont des rapports de réciprocité, comme ceux qui existent (peuvent exister) entre père et fils, mari et femme, etc. Le père n'est père que par rapport à son fils, qui, à son tour, est toujours le fils de son père, par rapport à son père ${ }^{7}$.

\section{7. « L'individu de groupe ». Les valeurs communes}

Selon Simondon, le groupe n'est pas fait d'individus réunis en groupe par certains liens. Sans exister isolément, les individus sont toujours individus de groupe, tandis que le groupe est toujours un groupe d'individus ${ }^{8}$. La polyvalence de la notion simondonienne d'« individu de groupe » rend très bien compte de l'indistinction entre l'individu et le groupe qu'implique la théorie du transindividuel. Somme toute, la communauté transindividuelle est un individu, un être ou un « organisme " collectif qui vit et agit comme un tout uni. Cela veut dire que la communauté transindividuelle a ceci de particulier que tous ses membres sont censés partager les mêmes valeurs. Suivant la définition qu'en donne Simondon, elle n'est rien d'autre que « la coïncidence mentale d'une pluralité d'hommes ».

Dans la mesure où l'autonomie de l'individu est mise en doute, on peut affirmer que l'avenir de l'individu est inséparable de l'avenir du collectif. D'un côté, l'individu ne meurt définitivement qu'avec la mort du collectif auquel il appartenait, en survivant en quelque sorte à sa propre mort physique. De l'autre côté, cette affirmation est nécessairement réversible : si le collectif meurt, l'individu ne peut pas rester sauf, il doit mourir avec lui.

\section{La communauté photographique est une communauté transindividuelle}

31 Dans la mesure où elle résulte d'une émotion partagée, la communauté qui s'instaure entre ceux qui co-apparaissent sur une photo est une communauté transindividuelle. Se faire photographier en présence de quelqu'un revient à reconnaître le fait d'avoir quelque chose en commun avec lui; en se prêtant simultanément à un acte photographique, les photographiés certifient le fait d'appartenir à la même communauté, ils signent, de leur propre volonté, un pacte d'amitié et d'égalité. Coapparaître sur une photo, c'est apparaître en tant que représentant d'une communauté, d'un groupe familial, politique ou d'un groupe d'amis. 

moyen ", de la réduire à une simple expression du mauvais goût du photographe, de sa position sociale. Selon Bourdieu, l'esthétique photographique fonctionne d'une façon inverse à l'esthétique kantienne : loin de combler l'abîme qui sépare les riches et les pauvres, elle accentue les différences sociales, en interdisant toute communauté, toute égalité entre les élites et les classes populaires. Mais la vision sociologique de la photographie est une vision foncièrement appauvrissante, qui passe sous silence son potentiel émotif'. Contrairement à l'affirmation de Bourdieu, la photo peut faire surgir de la communauté. Si, selon Barthes, chaque photographie est nécessairement un certificat de présence, on peut dire au même titre de chaque photographie de groupe qu'elle est un « certificat d'amitié ». Une fois qu'il est apparu sur une photo de groupe une fois qu'il est agrégé, une fois que le seuil est traversé - l'individu est condamné à faire partie du collectif. Dès ce moment, il lui est impossible de se séparer du collectif, impossible de retourner sur ses pas, de refaire à l'envers le chemin parcouru ; désormais, la vie individuelle s'associe à la vie et au destin du collectif.

\section{Le noircissement des photos}

Si la communauté photographique est transindividuelle et que les individus qui y appartiennent partagent les mêmes valeurs, la photographie sur laquelle le suspect apparait en présence d'un ennemi du peuple ne peut que témoigner de l'implication du suspect dans les affaires de l'ennemi. Le fait de co-apparaître sur une photographie avec un ennemi du peuple est une raison suffisante pour entamer les poursuites à l'encontre du suspect.

Le suspect fait partie d'une communauté où le « crime » de l'un est le crime de tous. $\mathrm{Si}$ les frontières de ma subjectivité coïncident avec celles du collectif auquel j'appartiens, je suis condamné à partager les crimes des autres, je ne peux pas rester " propre ». C'est pourquoi la communauté photographique, le fait d'être l'ami de l'ennemi, suffit à l'accusation.

C'est à partir de la photographie et plus largement de l'image que se construit la ligne de partage symbolique entre les fidèles et les subversifs. La photographie de groupe lieu stratégique de l'affrontement politique - peut servir à tester le suspect, car celui-ci a toujours la possibilité de changer de camp, de se démarquer de l'ennemi du peuple, de rompre le pacte d'amitié passé avec lui. La guerre moderne a besoin de cette extériorisation sans laquelle tout dépistage de l'adversaire se révélerait impossible. Le « suspect » est mis devant le choix qui laisse peu de place aux manœuvres : ou bien il se démarque de l'ennemi du peuple, le renie, ou bien il partage son sort. En détruisant la représentation de l'ennemi, le suspect l'exclut de la communauté, il fait en sorte que les crimes de l'ennemi du peuple ne soient pas les siens. 
Photographie 9

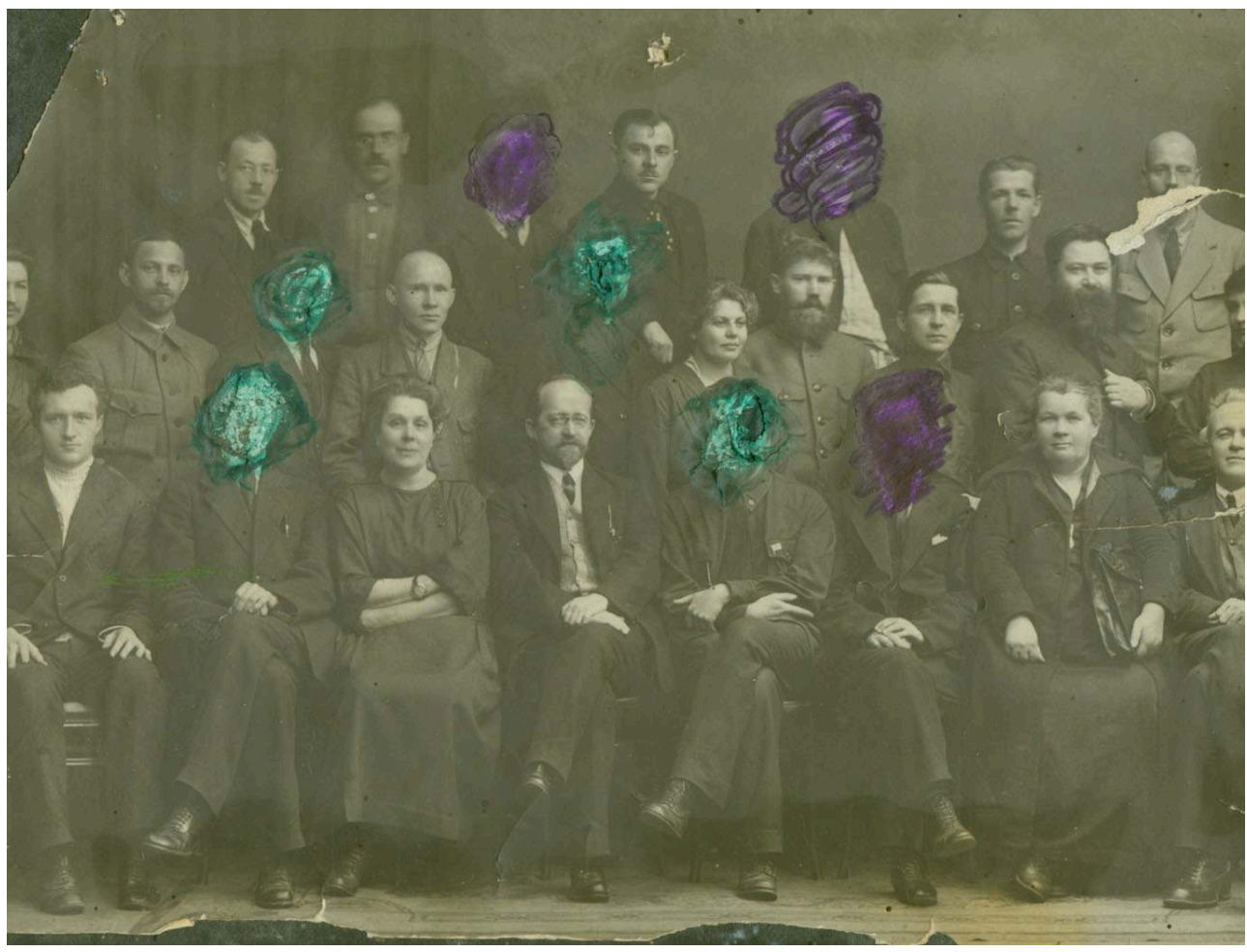

Photographie du dossier de Zoubarev, programme «ALGIR-2 »

Archives de Mémorial, Moscou

Ce reniement va de pair avec une «initiation ». À détruire la représentation de son ancien ami, l'individu partage les crimes de ses persécuteurs, devient l'un des oppresseurs, entre, contre son gré, dans leur communauté. Si les cas dont il s'agit sont une mesure d'intimidation, ils sont, pour les bourreaux, une façon de rendre coresponsable, de partager les crimes et la responsabilité. Cette situation renvoie directement à la théorie arendtienne du crime partagé et de la culpabilité collective. Le « suspect » ne se démarque du groupe auquel il appartenait auparavant qu'au prix d'en intégrer un autre, de devenir l'ennemi de son ami renié. La pratique qui s'apparente de plus près à l'élimination des représentations des ennemis, c'est celle des condamnations collectives, qui avaient généralement lieu au travail. Chaque collectif était obligé d'exprimer publiquement son attitude envers un ennemi du peuple, de prendre une position face aux actes d'un « espion » ou d'un « saboteur ». 


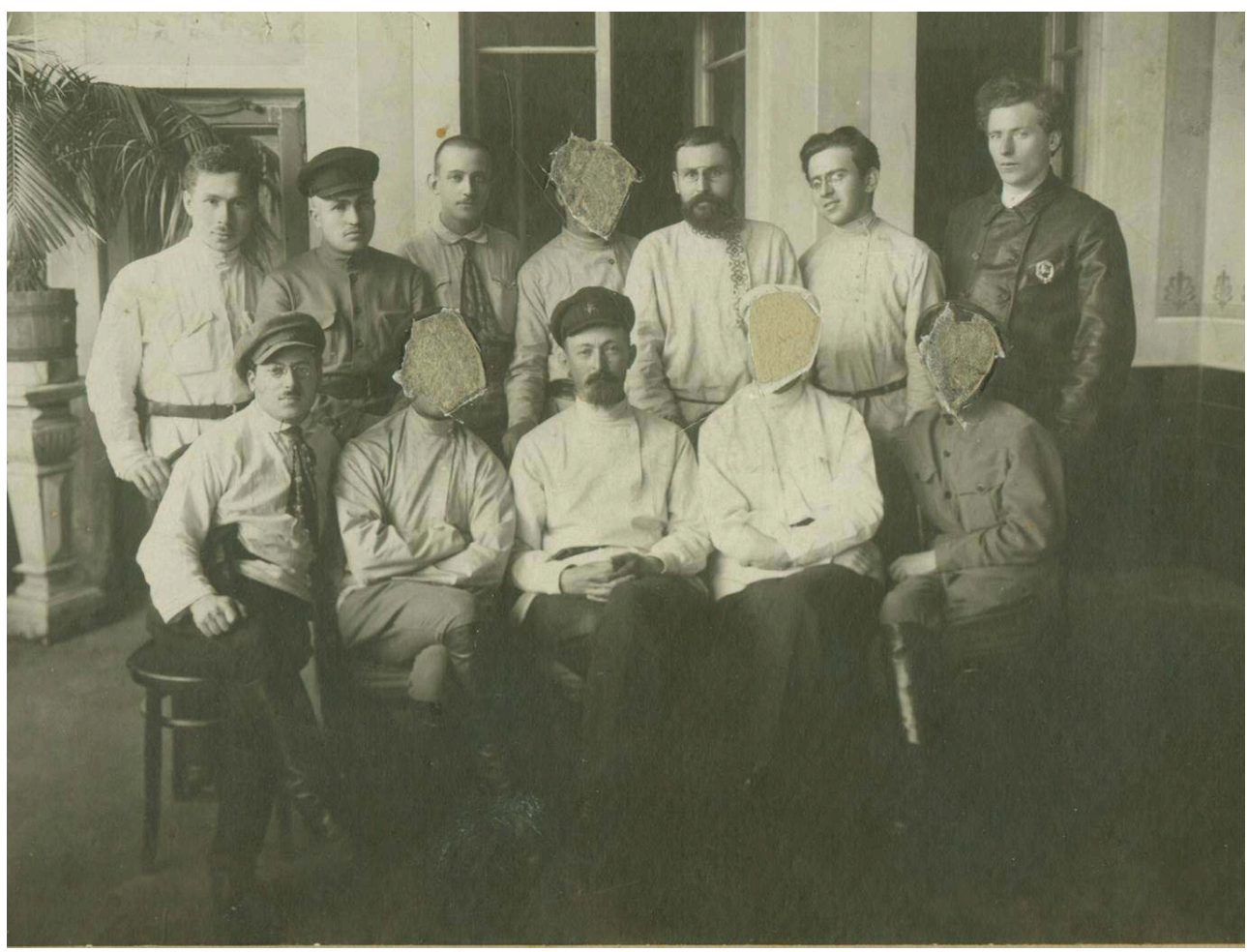

Dzerlinski entouré de ses collaborateurs. Photographie du dossier de Bérézine, programme «Photoscans ».

Archives de Mémorial

\section{Aloïs Riegl : le portrait de groupe est un « singulier pluriel ». La fraternité}

De toute évidence, la photographie de groupe comme appareil ne surgit pas du vide, étant précédée par le portrait de groupe pictural dont la tradition remonte à l'art hollandais du $\mathrm{Xv}^{\mathrm{e}}$ siècle. Les premiers portraits de groupe représentaient des individus qui se réunissaient en corporation en poursuivant un but commun. Ces individus formaient - surtout à l'âge d'or du portrait de groupe - une communauté essentiellement spirituelle, car les individus partageaient les mêmes valeurs religieuses ou politiques.

Selon l'historien de l'art viennois Aloïs Riegl, les portraits de groupe réunissent dans un espace pictural des individus parfaitement autonomes. Cependant, un portrait de groupe n'est en aucun cas une «somme» de portraits individuels, obtenue par leur simple juxtaposition. Ici, l'individualité du portraituré n'est pas celle d'un portrait simple, qui contribua largement à l'autonomisation de l'individu pendant la Renaissance. L'individualité de chacun est appareillée par un procédé pictural. Celui-ci fait obéir la singularité d'une apparition unique à un tout, fait surgir de la communauté au sein d'une réunion disparate et fait évoluer le préindividuel de chacun en direction d'un «milieu associé » (Simondon). La fonction du portrait de groupe est celle du déplacement et de la fusion symboliques, de façon que les individus représentés forment un tout spirituel. Selon Riegl, le portrait ouvre une nouvelle dimension, en 
dépassant « un dualisme entre objectivisme et subjectivisme ${ }^{10}$. Le portrait de groupe affirme une " individualité collective », transforme un groupe d'individus en " individu de groupe ».

39 Le portrait de groupe met en œuvre l'idée de la fraternité et de l'égalité de ceux qui y co-apparaissent. Chacun est représenté entre ses pairs et les rapports entre les personnages sont ceux de " coordination ». Il s'agit de rapports purement horizontaux d'où est absente toute trace de subordination. Cette façon d'apparaitre suspend les hiérarchies et les rapports de pouvoirs. C'est pourquoi la communauté dont il est question a une signification politique. Ce n'est pas un hasard si le portrait de groupe évite la scénographie, qui est toujours une hiérarchisation, une distribution de rôles sociaux. Le portrait de groupe ne se confond pas avec un tableau d'histoire ni avec une scène de genre, ayant un autre fondement ontologique. La mise en scène reste un procédé extérieur au portrait de groupe en tant que philosophie et en tant que genre. La scénographie ne doit pas embrouiller son pluralisme intrinsèque, ne doit pas introduire de la hiérarchie dans son espace égalitaire. Les individus doivent apparaître tels qu'ils sont, et non pas déguisés, non pas en acteurs. Cette description vaut également pour une photo de groupe. Sauf à cesser d'être ce qu'elle est, la photographie de groupe ne doit pas transformer les personnages en acteurs. Ils ne doivent pas jouer des rôles qui ne sont pas les leurs, ne doivent pas adopter des postures et des identités d'emprunt.

Politiquement parlant, le portrait de groupe affirme l'ontologie d'un «singulier pluriel ", nous fait retourner à la question de l'essence de la politique, la question du politique. Ici, nous sommes très proches de la tradition aristotélicienne, dont $\mathrm{H}$. Arendt et J.-L. Nancy se sont inspirés plus récemment pour penser le politique comme ce qui se trouve entre les individus. Le politique dans sa pureté apparaît là où se trouvent suspendus les rapports de pouvoir, les hiérarchies.

\section{Les aspects psychologiques et éthiques du noircissement des photos. Le reniement}

41 Il serait faux de voir dans le reniement un «simple effet » qui accompagne la terreur. Loin d'être un phénomène qui suit les purges, le reniement occupe la place centrale dans la structure de la terreur. Le reniement, en tant que destruction complète des rapports transindividuels, constitue le but ultime des disparitions politiques.

On connaît la thèse de Hannah Arendt selon laquelle la terreur avait pour objectif la destruction des classes politiques, en premier lieu du prolétariat. La réalité soviétique n'aurait rien à voir avec l'esprit communautaire dont se réclame la société dite " communiste». Le citoyen soviétique est plongé dans une existence purement individuelle, coupé de ses liaisons, réduit à une isolation presque complète ; la société soviétique est faite d'atomes humains privés de toute initiative. Le système totalitaire correspondrait à la destruction absolue des classes, c'est-à-dire des rapports qui se basent sur la communauté des intérêts de ceux qui y appartiennent. Pourtant, Arendt va plus loin : le totalitarisme brise non seulement les classes mais plus largement les rapports sociaux et même familiaux. Mais la défaite des rapports familiaux correspondrait au débordement par la terreur de ses cadres sociologiques, à un déchaînement, une crise de folie où la terreur perd de sa logique sociale. En réalité, cette «folie» de la terreur, cette tache aveugle dont parle Arendt peut signaler que 
l'épicentre de la terreur se trouve ailleurs que dans la vie sociale, quoique celle-ci en soit gravement atteinte. Arendt voit dans la destruction de la famille l'un des effets de la terreur, son débordement et sa " pathologie » (la maladie de la maladie) tandis qu'il faudrait inverser l'hypothèse pour y voir la manifestation la plus conséquente de la terreur. La terreur fait se dissoudre les rapports transindividuels, ceux qui s'opposent aux rapports interindividuels (rapports sociaux qui obéissent à la loi du profit et qui sont foncièrement hiérarchiques). Les rapports transindividuels sont ceux d'affection, ils se détachent du social et vont à l'encontre du social. Ils reposent sur l'idée du désintéressement, sont incompatibles avec aucun profit, se construisent comme la négation du profit.

C'est donc dans la famille que la logique de la terreur se met à nu. Comme les rapports transindividuels culminent dans la famille, la famille se trouve dans l'épicentre de la terreur et demeure son enjeu stratégique. La famille est le dernier refuge de l'éthique, de ce qui résiste encore à la politisation, à la totalité de la guerre, à l'ubiquité de l'opposition politique ami/ennemi. La sphère privée ne doit pas rester hors de la sphère politique : si j'aime mon proche, fût-ce « en privé », il me sera difficile de le haïr en tant que mon ennemi politique (hostis publicus). Comme en témoigne l'expérience soviétique, la distinction faite par Schmitt qui veut séparer le privé et le public comme espaces respectifs d'amour et de haine est hautement artificielle. Contrairement à ce que prétend Schmitt, il est impossible d'aimer un ennemi en privé et le détester politiquement. Au besoin, l'homme soviétique doit laisser entrer la guerre sous son toit, tracer la ligne de front au sein de sa famille. Un bon citoyen doit veiller à son prochain quel qu'il soit, il doit savoir distinguer en lui des signes inquiétants et, au besoin, déceler en lui un ennemi caché. S'il le faut, les enfants seront les premiers à renier leurs parents, à leur déclarer la guerre. La ligne de front peut passer entre les parents et les enfants.

\section{Rapports entre parents et enfants}

La fameuse déclaration de Staline, « le fils n'a pas à répondre pour son père ${ }^{11}$ ", reprise par la presse soviétique et transformée en slogan, est à interpréter comme incitation au reniement, car elle impose comme naturelle l'absence de rapports symboliques entre le père et le fils. La dette filiale envers le père apparaît alors comme inexistante. Grosso modo, le fils qui n'a pas à répondre pour son père, cesse d'être le fils de son père, cesse d'être son « héritier » (Derrida). On ne peut se libérer de la responsabilité qu'à ce prix. Ne pas avoir à répondre pour son père, c'est reconnaître la culpabilité de son père, c'est renier sa communauté avec lui, se démarquer de lui, l'abandonner.

Si le caviardage des photographies est une forme de l'abandon, la lettre de reniement en est une autre, officialisée par le régime et sans doute la plus répandue. L'une et l'autre ont ceci en commun que l'enfant condamne son père ou sa mère pour se ranger $\mathrm{du}$ «bon » côté. Il s'agit d'une prise de position publique : l'enfant trace une ligne de front entre lui et son père, le déclare son ennemi et se range du côté de ses persécuteurs.

Il y a des lettres de reniement qui sont très bien écrites. À les lire, on a l'impression que la rupture se produit avec facilité. Mais cette facilité est toujours apparente. Dans la plupart des cas, ce sont des lettres de reniement « officielles ", écrites sous la dictée. La douleur causée par la rupture du rapport transindividuel est extrêmement aiguë - non 
seulement pour celui qui est renié, mais aussi pour celui qui le renie : si les frontières de ma subjectivité s'étendent vers celles du collectif auquel j'appartiens, tout reniement est nécessairement autodestructif. Je renie une partie de moi-même, je deviens mon propre ennemi. Mis devant un choix impossible, la majorité des enfants vivent un véritable clivage au sein de leur « moi », ils sont désorientés et écrasés.

Il y avait des cas où un enfant écrivait à ses parents arrêtés afin de savoir s'ils étaient vraiment coupables, s'ils avaient effectivement commis les crimes dont on les accusait. Alors, c'étaient les parents qui étaient mis devant un choix douloureux, car ils savaient que leur enfant ne pouvait pas vivre dans la société soviétique sans renier son proche ennemi du peuple, qu'il serait persécuté.

\section{Rapports mari/femme}

Les rapports conjugaux peuvent être des rapports à forte réciprocité et, de ce fait, devenir rapports symboliques. Dans la mesure où les rapports mari/femme sortent du cadre sociologique - cessent d'être des rapports d'échange, se libèrent des marques de pouvoir et de dépendance - ils acquièrent un caractère transindividuel. Loin d'être la preuve de l'incompétence politique des femmes, ou bien de leur indifférence à l'égard de la politique, le fait qu'une femme a rarement des convictions politiques différentes de celles de son mari témoigne de l'étroitesse du rapport transindividuel entre les conjoints. Contrairement à ce que prétend Schmitt, il est impossible d'aimer un ennemi en privé et le détester politiquement. Comme en témoigne une série de mesures répressives dirigées contre les épouses des ennemis du régime, les pouvoirs soviétiques et Staline personnellement étaient pleinement conscients de cette impossibilité et de l'étroitesse "politique " des rapports entre les époux. Ces mesures avaient le même objectif de faire renier leurs conjoints aux femmes.

D'un côté, c'étaient des mesures d'intimidation et d'oppression. L'un des articles du Code pénal, ajouté à l'initiative personnelle de Staline, prévoyait l'arrestation des conjointes des ennemis du peuple. Si elles n'étaient pas privées de liberté, elles étaient " chassées de chez elles, renvoyées de leur travail, privées de leur ration ou de leurs droits civiques ${ }^{12} »$. On pratiquait également des retenues sur salaire, on gelait les épargnes, on augmentait le loyer. De l'autre côté, des mesures d'encouragement ne manquaient pas non plus : ayant droit de prendre l'initiative unilatérale du divorce, les femmes des ennemis du peuple y étaient encouragées par le coût réduit de la procédure, qui fut ramené de 500 roubles à 3 roubles quand on divorçait d'un " ennemi ${ }^{13}$ ». Tombée de Charybde en Scylla, la femme de l'ennemi du peuple doit renier son conjoint. Si le reniement constitue la règle et le but ultime de la terreur, les cas où le reniement n'a pas eu lieu doivent être tenus pour exceptionnels.

Deux photos de famille ci-dessous présentent un grand intérêt. La première est celle d'un couple familial (11) où la représentation du mari a été découpée. Nous possédons des renseignements assez précis sur cette famille, sachant leur nom et la date de l'arrestation du mari (1937). 
Photographie 11

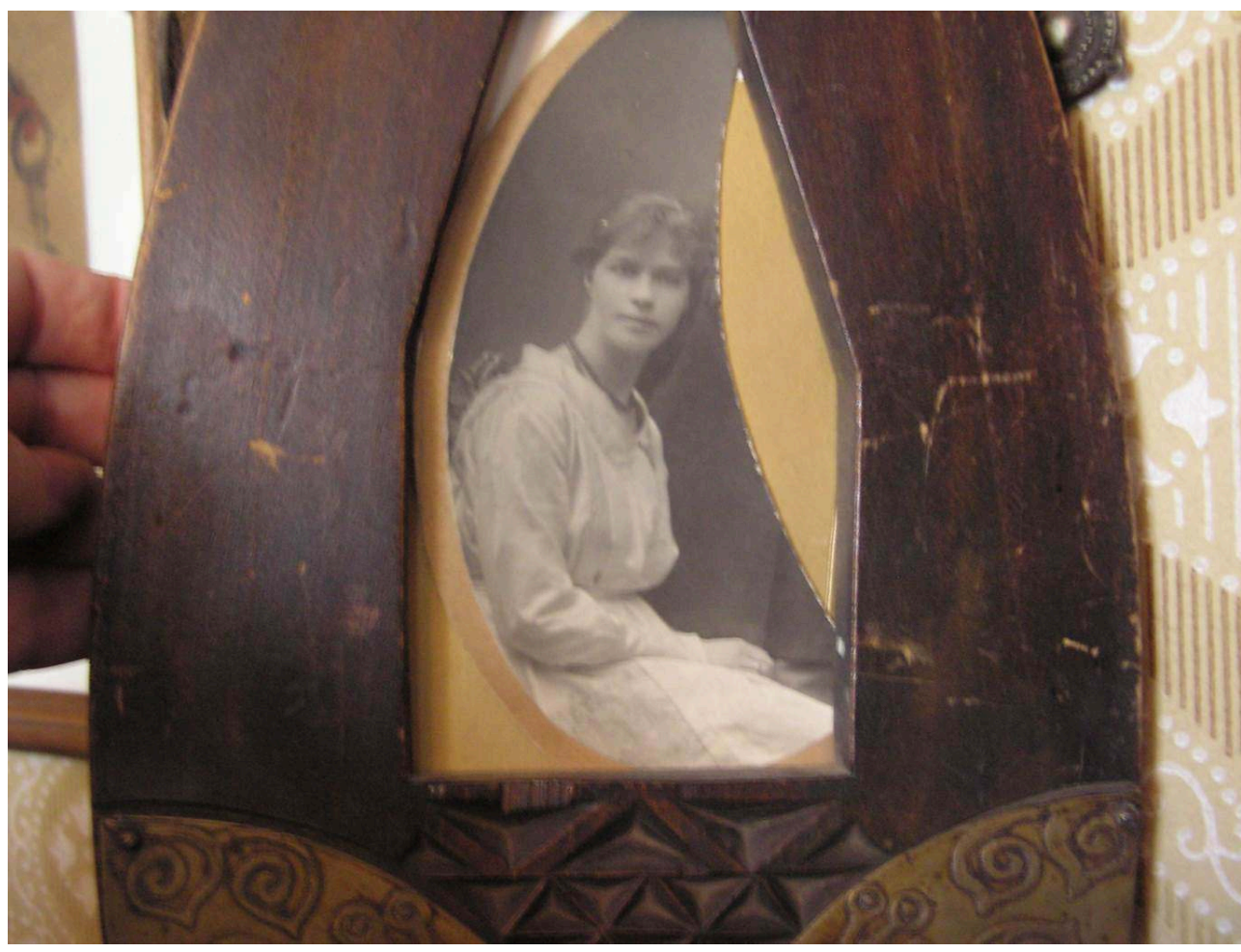

Un couple familial. La représentation découpée est celle du conjoint arrêté en 1937.

(c) Archives de Mémorial, Moscou

51 Par contre, nous ne savons rien de l'autre photographie (12), qui se trouve à Mémorial parmi les photos anonymes, qui ne sont pas classées dans les albums de famille. 
Photographie 12

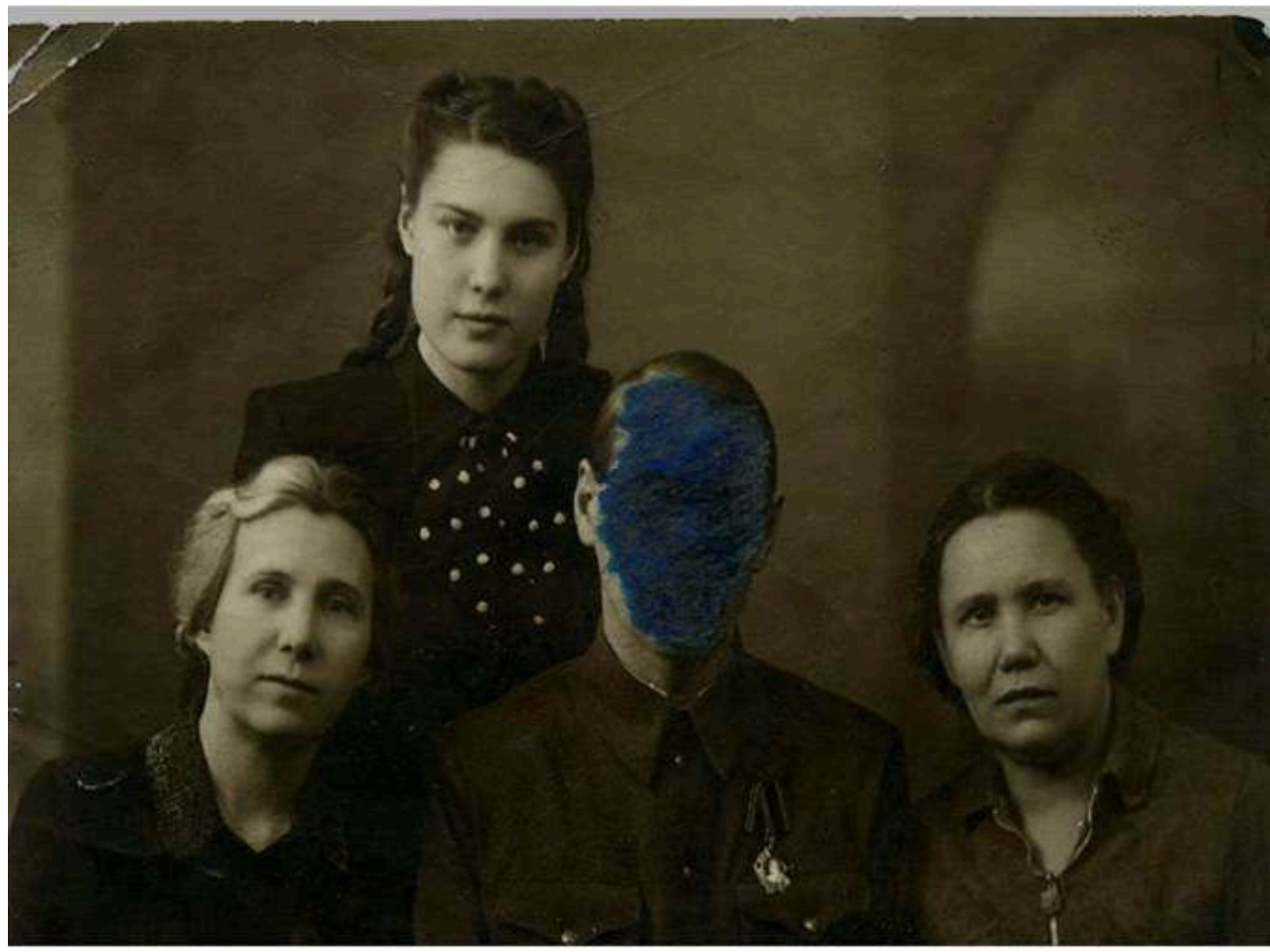

Portrait de famille avec un jeune officier au visage " caviardé ». Photographie qui se trouve parmi les photos anonymes n'ayant pas d'assignation aux albums familiaux.

(c) Archives de Mémorial, Moscou

52 Cette photographie est sans doute l'une des plus intéressantes parmi celles qui se trouvent à notre disposition. De toute évidence, il s'agit d'une photo qui représente des parents proches. Les questions qui se posent sont les suivantes: de quelle parenté, exactement, s'agit-il ? Qui a attaqué la représentation de l'ennemi ? Cette photographie frappe le spectateur par la façon dont on attaque le visage de l'officier, à savoir, la violence de cette attaque.

\section{L'effort fait pour éviter le reniement}

Comme il a été remarqué tout à l'heure, la forme de l'attaque iconoclaste peut varier. Selon les circonstances, la punition symbolique infligée à la représentation de l'ennemi peut être plus ou moins dure.

Il y avait des cas où l'individu qui se savait menacé et se trouvait en présence d'un cas de conscience douloureux, voulait trouver un compromis entre sa sécurité et sa bonne foi. Dans ces conditions, l'acte iconoclaste est à peine perceptible, étant plutôt effectué pour la forme.

C'est ce qu'on voit sur la photo suivante représentant des délégués communistes en compagnie de Staline, où huit visages sur trente sont rayés en croix avec un crayon (13, 14). 
Photographie 13

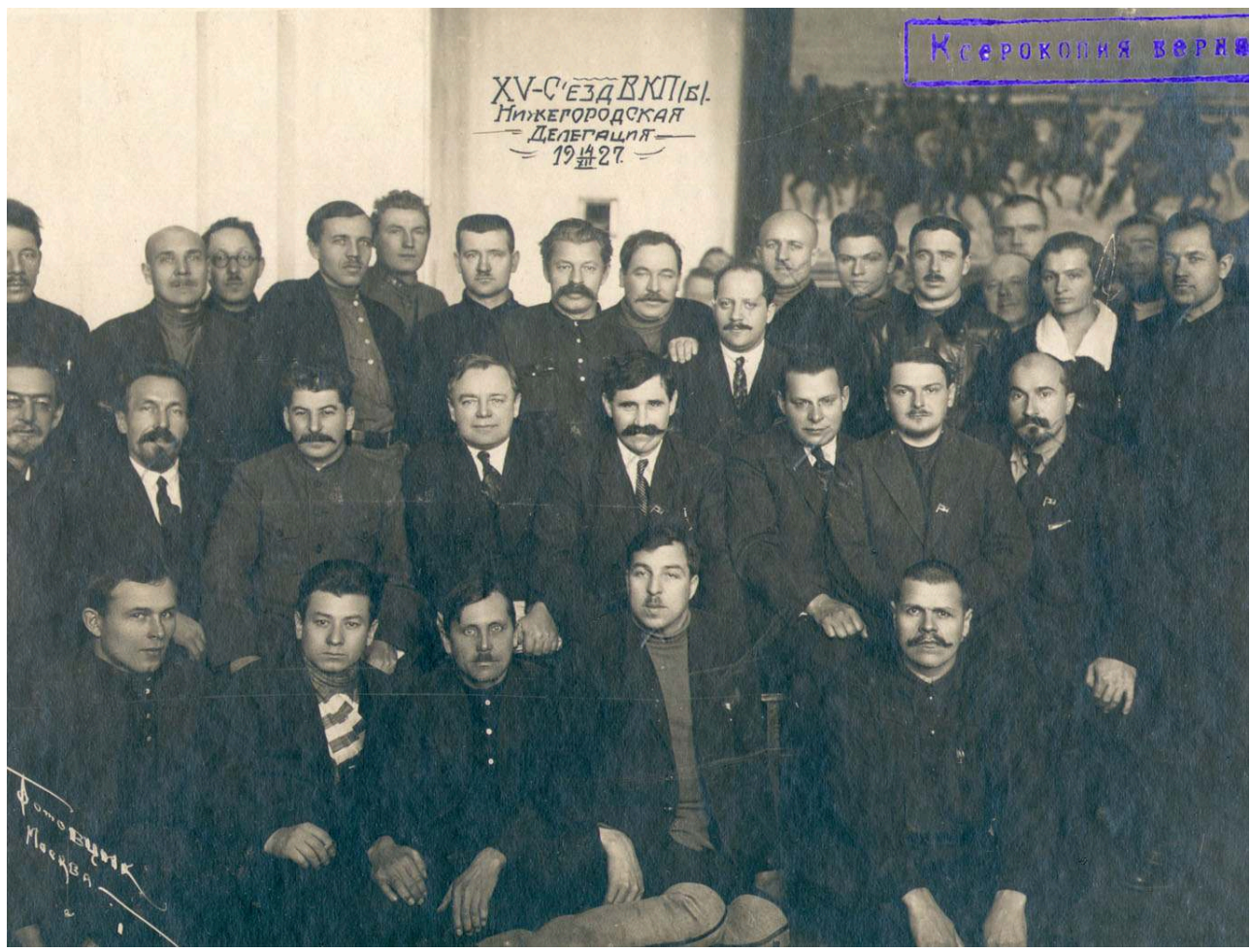

Anonyme, La délégation de Nijni-Novgorod avec les membres du gouvernement. Le xve congrès du VKP(b). Moscou, le 15 mars 1927. Archives du Comité régional du Parti Communiste de Nijni-Novgorod / Fonds 7853 , dossier 394, inventaire 1.

(C) Archives du Comité régional du Parti Communiste de Nijni-Novgorod 


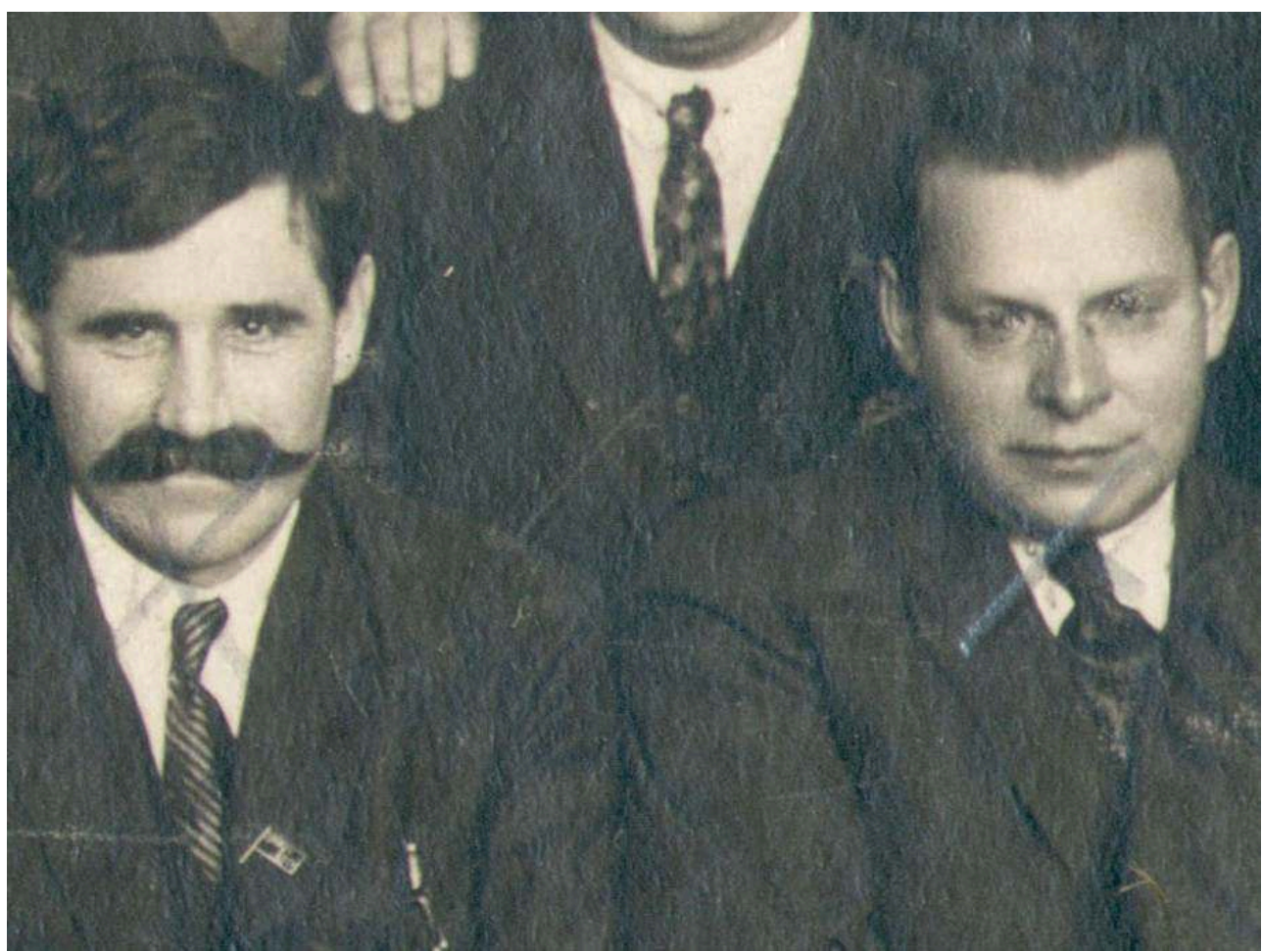

Anonyme, La délégation de Nijni-Novgorod avec les membres du gouvernement. Le xve congrès du VKP(b). Moscou, le 15 mars 1927. Archives du Comité régional du Parti Communiste de Nijni-Novgorod / Fonds 7853, dossier 394, inventaire 1 (fragment)

(c) Archives du Comité régional du Parti Communiste de Nijni-Novgorod

Il est toutefois douteux que ce compromis soit efficace. Celui-ci s'inscrit contre le système des disparitions politiques, dont l'objectif est justement d'exclure tout compromis, toute neutralité, d'étouffer dans l'œuf toute tentative de résistance passive. En effet, la guerre moderne met en cause la notion même de neutralité. Si le conflit perd son caractère local pour se répandre sur l'ensemble de la population, la neutralité doit être dénoncée en tant que déguisement, simulation dont profite l'ennemi pour éviter la répression. À la différence de la guerre classique, la guerre moderne qui se veut absolue se montre hostile à l'absence d'hostilité.

Ici, le «suspect " cherche à contourner l'épreuve, à l'escamoter, comme s'il ne comprenait pas ce que lui voulait le pouvoir. Mais l'insuffisance de la punition infligée à l'image de l'ennemi se retourne contre le punisseur : s'arrêter à mi-chemin et ne pas noircir le visage de l'ennemi du peuple revient à être présent sur une photo à côté de celui-ci, à se reconnaitre comme appartenant à la même communauté que lui et, de ce fait, à partager ses « crimes ». Toute manœuvre entreprise en vue d'éluder le reniement est destinée à l'échec.

59 Parfois, les proches d'un ennemi du régime essayaient de garder sa photographie - ce qui veut dire garder les rapports affectivo-émotifs avec lui - et, en même temps, d'éviter le danger qu'ils couraient du fait de la garder.

Bien qu'elles aient un aspect différent, les photos 15 et 16 sont tirées du même cliché. Il s'agit donc de la même photographie, la différence entre deux tirages consistant en ce que les parents de la personne représentée ont supprimé, en le noircissant, l'uniforme du général de l'armée impériale. 


\section{Photographie 15}

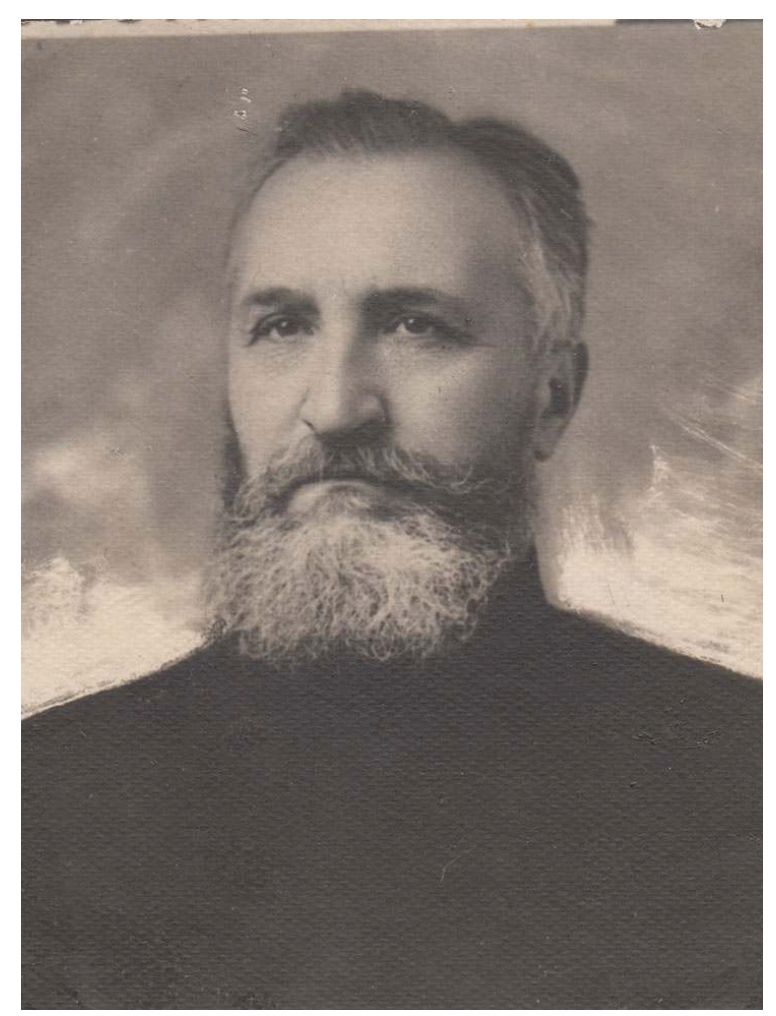

Photographie du dossier de Makchéev. Programme Le dernier témoin, Archives de Mémorial, Moscou. (c) Mémorial, Moscou 


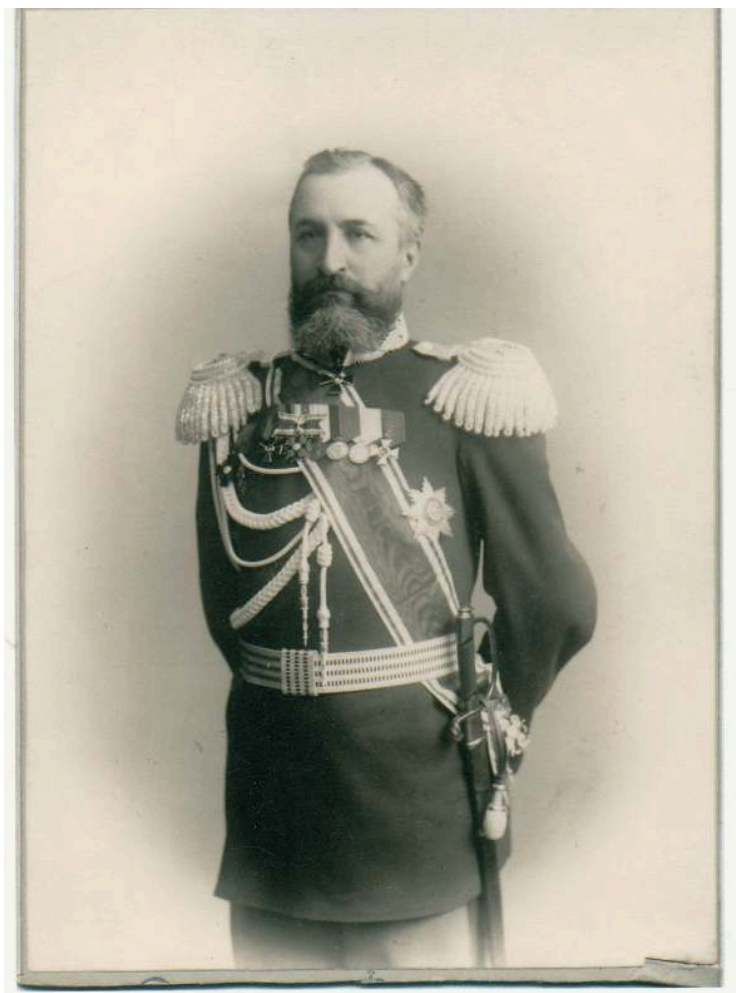

Le général F. Makchéev. Photographie du dossier de Makchéev. Programme Le dernier témoin, Archives de Mémorial, Moscou

(C) Mémorial, Moscou

Si l'on retourne à la photo commentée tout à l'heure, on voit que celui qui a commis l'attentat iconoclaste ne cherchait pas de compromis, bien que la personne qui l'a fait dut appartenir à la même famille et, probablement, fut présente sur la photographie.

\section{La signification du visage}

Dans le contexte qui est le nôtre, on ne saurait passer sous silence la problématique du visage. La question est de savoir si c'est bien le visage qui sert d'intermédiaire au rapport transindividuel. On sait que, selon Emmanuel Levinas, la loi éthique qui transcende le monde des phénomènes se révèle dans l'épiphanie du visage humain. Le visage serait un non-phénomène par excellence, il s'offrirait à mon moi sans condition. Ce point de vue a été critiqué par Jacques Derrida. Si Derrida fait sienne la logique de la trace, il émet néanmoins des doutes sur le visage en tant que support de cette logique, sur l'« efficacité » de son épiphanie comme "moyen de transmission» de la relation éthique. Selon Derrida, avec sa philosophie du visage, Levinas tombe dans le panneau d'une " urgence empirique », de la métaphysique de la présence. Le visage, en raison de sa phénoménalité, ne peut pas être la trace d'autrui.

Gilles Deleuze lui aussi met en question la singularité et l'immédiateté du visage. Selon lui, le visage est social avant d'être individuel, en neutralisant tout ce qu'il y a d'individuel, de singulier, de rebelle. Dans son visage, autrui s'absente complètement. Deleuze récuse le visage comme universel pour le considérer comme sous-produit du 
christianisme ; le face-à-face est une structure qui naît d'une configuration de pouvoir dans les sociétés chrétiennes.

Marie-José Mondzain, qui entreprend une étude sur l'imaginaire du visage et de la frontalité, arrive aux mêmes conclusions que Derrida et Deleuze. La frontalité comme mode privilégié du rapport à autrui ne s'instaure qu'avec le christianisme. À leur tour, ceux qui sont exclus de la communauté sont censés ne pas avoir de visage. Le cas des Juifs est le plus exemplaire. Privés de visage, les Juifs sont vus toujours « de profil ».

65 Ce deuxième point de vue me parait justifié. Le visage n'est pas universel et les rapports qu'il véhicule sont plutôt des rapports sociaux. Dans la majorité des cas, le reniement ne s'accompagnait pas de privation de visage. Ce n'est donc pas le visage qui se trouve à l'épicentre des coups iconoclastes, mais un autre objet, un «objet » non phénoménal qui véhicule le rapport émotivo-affectif que le visage, paradoxalement, ne véhicule pas. Celui qui s'attaque à la représentation cherche à détruire la communauté photographique entre lui et l'ennemi du peuple - la communauté photographique qui est en même temps une communauté politique. Tout porte à conclure que le rôle que Levinas attribue au visage peut être confié à la photographie en tant que trace de l'autre, ou plus précisément, à l'essence de la photographie, le photographique qui ne se constitue ni comme présence pure ni comme absence pure et qui crée et véhicule le rapport transindividuel. C'est pourquoi la privation de visage n'était jamais un but en soi. Elle a lieu seulement dans le cas où la personne va jusqu'au bout, fait de l'autre son ennemi personnel. C'est une façon très radicale de refuser la communauté.

\section{Les autoreniements}

Dans la mesure où l'on renonce au substantialisme, en affirmant, avec Simondon, que la subjectivité n'a pas de limites bien précises et évolue dans un milieu transindividuel, il faut aller plus loin et dire que la ligne de front qui sépare l'ennemi et l'ami peut passer à l'intérieur de l'individu. D'où le fait que la suspicion du citoyen peut porter sur une partie de lui-même: il doit rester vigilant, en garde contre lui-même tout en distinguant des idées subversives dans sa conscience de classe. Un bon citoyen communiste doit savoir se méfier de lui-même, veiller à ce que des idées subversives ne s'emparent pas de lui à son insu. Tout comme il renie son prochain, il doit constamment se renier lui-même, distinguer et couper à la racine des éléments de droite ou trotskistes au fond de sa conscience politique. Le communiste lutte contre la subversion qui l'attaque de l'intérieur avec les armes de l'introspection et de l'autocritique.

67 L'autocritique est à considérer comme un acte d'autoreniement, et c'est ici qu'il faut chercher la clé à cette importance qu'a la confession publique sous Staline. Contrairement à ce qu'il peut paraitre, le stalinisme encourage l'écriture de soi, les différentes formes de l'aveu et de l'autocritique.

Chaque repentant est censé faire ses confidences oralement, en présence du public, et par écrit, soit dans son journal intime (ce qui ne signifie d'ailleurs pas « en privé»), soit sous forme d'autoreprésentation critique qu'il soumettait au comité du Parti.

Bien entendu, tout comme le biffage des photographies, l'autocritique fonctionne à l'époque de la terreur comme stratégie d'escamotage et de prévention, car elle permet 
- si faible que soit cet espoir - de se prémunir contre des accusations éventuelles et de se garantir contre la terreur.

70 La brutalité des purges ne doit pas empêcher de voir la complexité des mécanismes éthiques qui se cachaient au fond de la distinction dite " politique » entre les amis et les ennemis. En dénonçant les éléments subversifs au fond de lui et en reniant ces éléments, l'individu se purifie politiquement et se range du «bon» côté du front politique. Il ne s'agissait pas, bien entendu, de voir dans cette autocritique la manifestation de quelque sincérité, mais la manifestation de la loyauté. De toute évidence, il s'agissait d'une prise de position forcée, d'un acte de violence à l'encontre de l'individu qui lui faisait se trahir lui-même. L'individu ne survivait qu'à condition de cesser d'être lui-même, de se dénoncer, se trahir, se ranger contre lui-même du côté de ses propres oppresseurs. L'individu qui a cédé à la pression et a manifesté de la peur se signalait par là comme bon citoyen: celui qui peut se trahir soi-même trahira les autres. Toujours est-il que les responsables de la terreur savaient que la volonté éthique ne va jamais sans volonté politique. L'individu qui permet qu'on lui ôte la volonté éthique est aussi apolitique que possible.

\section{Les photographies portant des inscriptions}

71 L'intérêt de trois photographies qui se trouvent ci-dessous consiste dans le fait de porter des commentaires manuscrits, les traces du «travail » des enquêteurs du NKVD (la police secrète stalinienne).

La première photographie (17) représente un groupe de riches marchands, tous régents d'un orphelinat à Nijni-Novgorod (c'est un portrait de groupe classique qui fait penser aux portraits de régents par Hals). On peut y voir les flèches ainsi que les inscriptions qui nous renseignent sur les personnages (leur nom, leur condition sous l'ancien régime...). 
Photographie 17

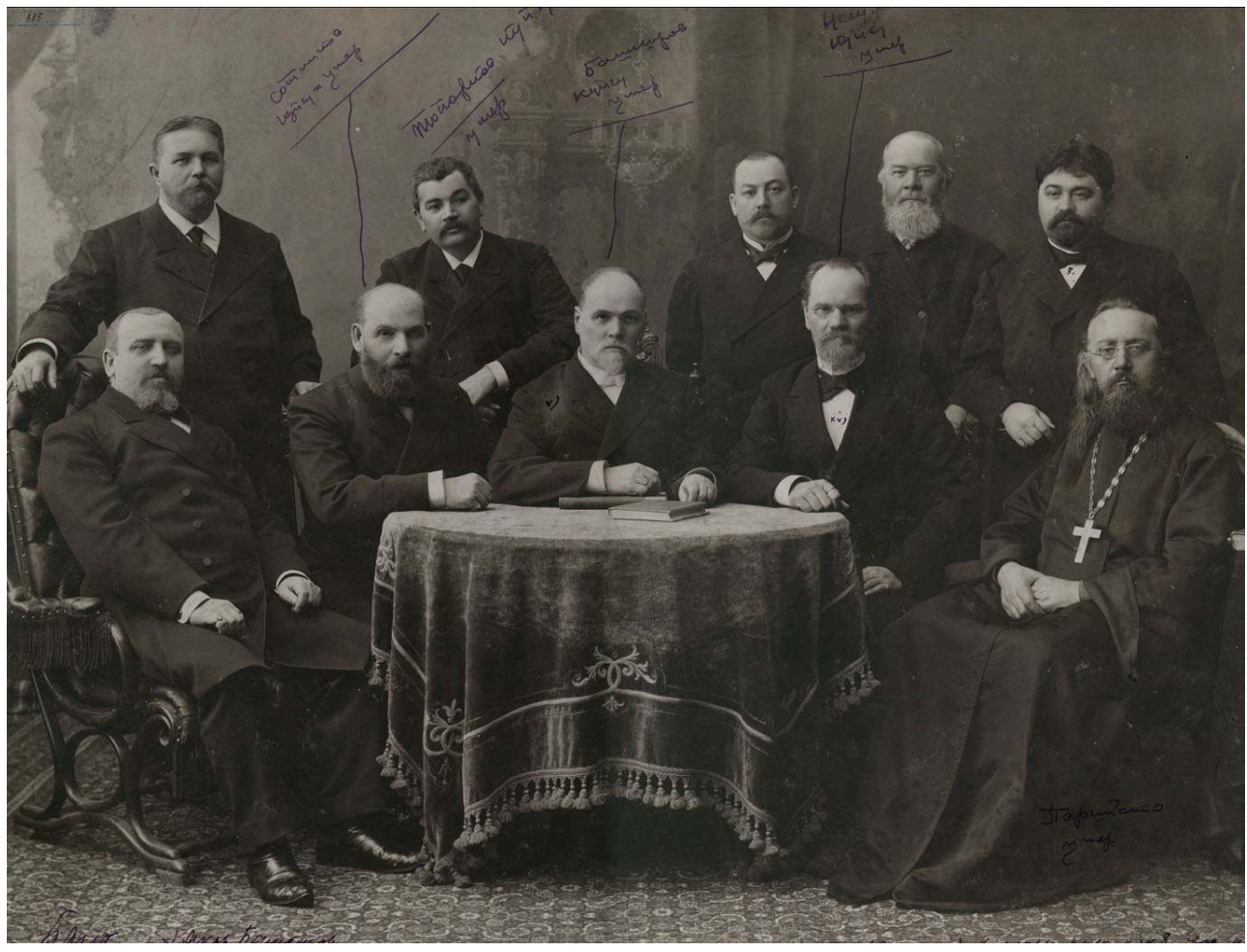

L'archevêque Nazaire et le groupe des bienfaiteurs de l'orphelinat Kutaissov. 1913. Archives audiovisuelles de Nijni-Novgorod / Inventaire 1.1/8, 66-3060.

(c) Archives audiovisuelles de Nijni-Novgorod

73 Deux autres photos $(18,19)$ sont prises peu avant la Révolution (en 1913) et représentent des policiers. Nous apprenons, des commentaires manuscrits laissés par les NKVD, si le personnage est mort ou bien en vie; dans le cas où il n'est pas mort, son lieu de résidence, son poste dans la police d'avant la Révolution ainsi que les actes contre-révolutionnaires qu'il a commis (par exemple, une des inscriptions dit que le commissaire Pétrov a pris part à l'écrasement de l'insurrection de Sormovo en 1905). 
Photographie 18

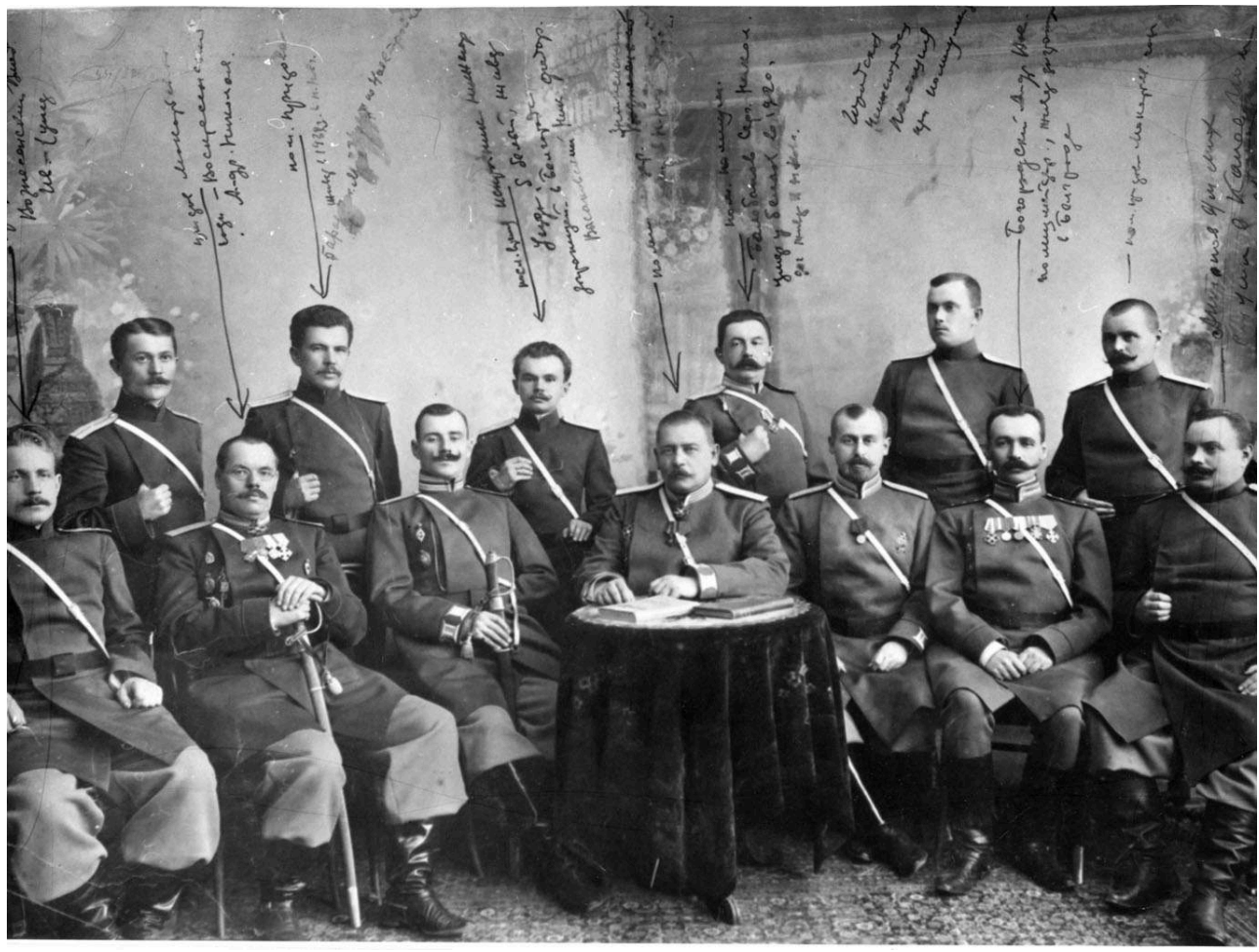

Anonyme. Un groupe de policiers de Nijni-Novgorod avec le chef de police A. Znamenski. Archives audiovisuelles de Nijni-Novgorod / Inventaire 726.

(c) Archives audiovisuelles de Nijni-Novgorod 


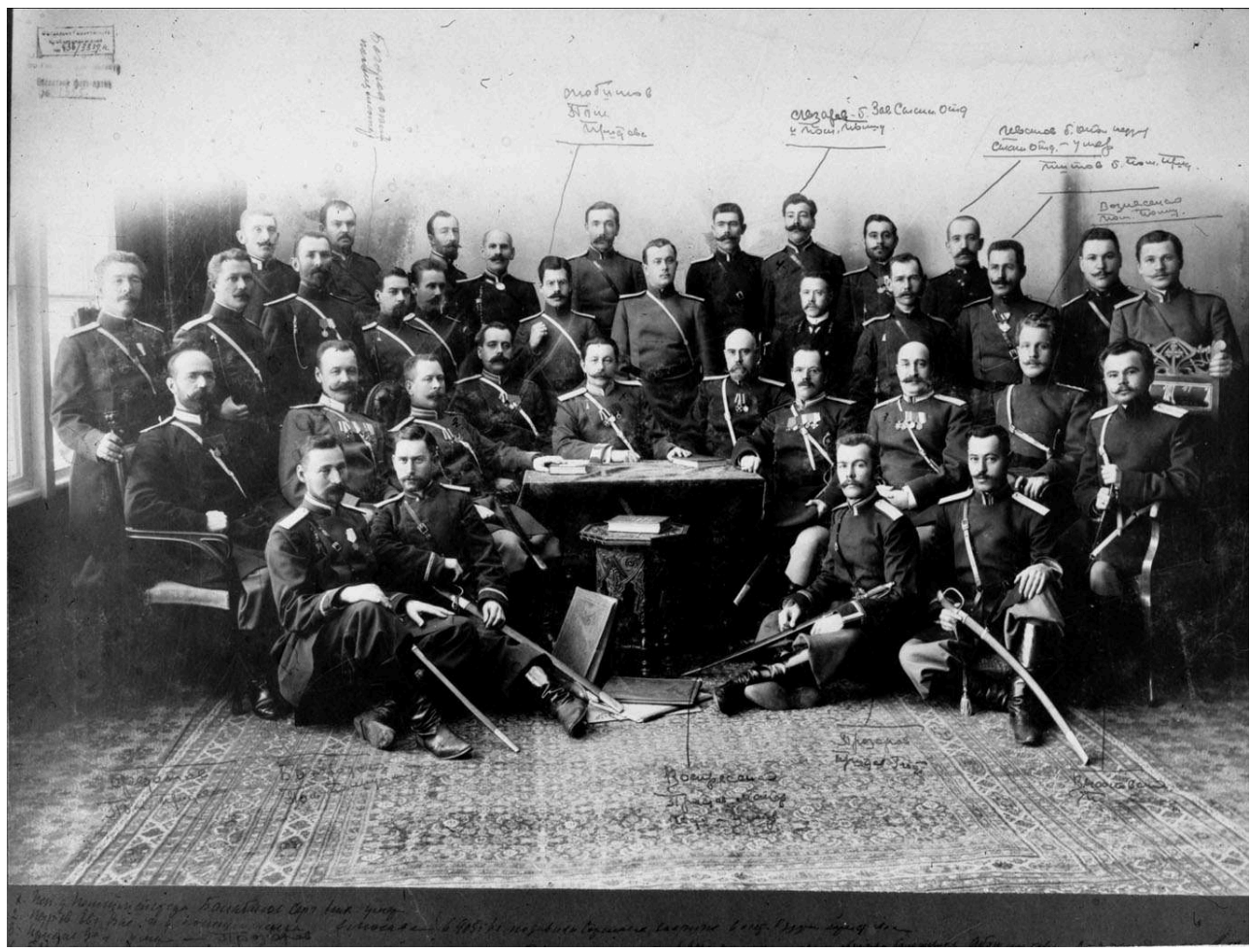

Anonyme. Police. Deux groupes. Archives audiovisuelles de Nijni-Novgorod / Inventaire 300.

(C) Archives audiovisuelles de Nijni-Novgorod

74 Il est évident que les photographies qui portent des inscriptions constituent un groupe à part, car les traces qu'elles gardent appartiennent à une autre catégorie et apparaissent dans un autre contexte (elles ne sont pas laissées par les victimes, mais par les persécuteurs). En même temps, elles semblent témoigner du même pouvoir de l'image photographique.

Qu'est-ce qui, exactement, intéressait les enquêteurs? S'agissait-il pour eux de reconnaître ceux qui figuraient sur la photographie? L'explication selon laquelle ces photos ont été utilisées pour reconnaître les ennemis du régime semble aller de soi. Pourtant, l'examen plus approfondi du cas montre que l'objectif de la reconnaissance ne se trouvait pas au cœur de cette « utilisation » des photographies.

76 En effet, la reconnaissance n'est possible qu'à condition de connaître au préalable celui qu'on re-connaît. Il est impossible de reconnaître sans connaître, et la reconnaissance ne va jamais sans connaissance, sous-entendant logiquement une reprise, une réitération, une coïncidence d'un savoir préliminaire avec une donnée sensible. La reconnaissance et l'identification policière reposent entièrement sur cette préconnaissance $^{14}$. C'est pourquoi l'usage policier de la photographie reste, à peu d'exceptions près, un usage de prévention dirigé contre une "récidive ", ce qui fait que la photographie judiciaire a un champ d'application très étroit. Comme le montre Christian Phéline, l'entreprise de Bertillon fut un échec ${ }^{15}$. Bertillon était un grand enthousiaste de son affaire, mais ses espoirs se sont effondrés justement en raison de l'efficacité fort limitée de la photo pour les objectifs de reconnaissance. 


\section{La photographie signalétique}

77 La question qui préoccupe Alphonse Bertillon est d'identifier le criminel. Il cherche à faire travailler le potentiel documentaire de la photographie, à libérer la photographie de tout ce qu'elle a de conventionnel et d'artistique. Si l'acte photographique est purifié de tout ce qui peut le «contrarier", la reconnaissance peut regagner en efficacité. L'objectif de Bertillon comme photographe est contraire à celui de son contemporain Nadar et, plus généralement, à l'effort de l'artiste, qui transforme le réel, qui fait un travail de symbolisation.

78 Il y a des gens naïfs qui s'étonnent et s'indignent en voyant jusqu'à quel point les enlaidit la photo d'identité dans leur passeport. Mais la photo dans le passeport, est justement une photo signalétique qui a pour objectif de dépouiller l'individu de tout ce qui le rend beau et empêche de le reconnaître.

79 On voit que, tout pratique qu'il est, l'usage judiciaire de la photographie n'est pas sans fondement théorique. Il repose sur le présupposé que la photographie est une empreinte. Somme toute, Bertillon met l'accent sur la capacité de la photographie de fixer le singulier, l'unique, l'individuel, tout comme le font, au $\mathrm{xx}^{\mathrm{e}}$ siècle, les partisans de la théorie dite "indicielle» de la photo, les esthéticiens comme R. Krauss, $\mathrm{Ph}$. Dubois, H. Vanlier. De tous les théoriciens de la photo comme empreinte, indice ou punctum, c'est Alphonse Bertillon qui va jusqu'au bout de la logique. La photographie judiciaire est purement informationnelle, dépouillée de son pouvoir de généraliser, qui empêche l'identification. Identifier, reconnaître une personne, c'est toujours l'individualiser, particulariser, isoler, séparer des autres représentants de l'espèce.

\section{L'usage de la photographie par le NKVD n'était pas un usage signalétique}

Or l'usage de la photographie par le NKVD n'avait rien à voir avec l'identification comme l'avait conçue Bertillon. Les policiers staliniens ont utilisé la photographie autrement que ne l'avait fait Bertillon, n'ayant pas pour but de reconnaître qui que ce soit. Ce qui les intéressait et ce dont ils étaient pleinement conscients, c'était le pouvoir, propre à la photographie de groupe, de construire des rapports affectivoémotifs entre les individus photographiés, de créer un « individu de groupe ».

81 Si la méthode exacte du travail sur ces photographies nous reste inconnue, on peut néanmoins faire une hypothèse relative à la façon dont elles étaient utilisées. Saisies au moment de l'arrestation d'un " coupable », elles lui auraient été présentées par la suite lors des interrogatoires. Le coupable aurait été interrogé sur l'identité de ceux qui l'entouraient sur telle ou telle photo de groupe. Dans les conditions de la guerre que l'État menait contre ses ennemis intérieurs, leur co-apparaître sur la photographie, le fait d'avoir partagé le même lieu et le même temps avec le coupable était suffisant pour garantir leur culpabilité. Pour le dire en termes qui sont ceux de Simondon, l'identité qui intéressait les enquêteurs n'était pas individuelle, mais une " identité de groupe ».

82 Cet usage des photographies par les gens du NKVD n'a rien de signalétique ni d'anthropométrique. Bien qu'il puisse ressembler à l'usage " policier ", il ne se confond pas avec lui. La question d'identité et des moyens qui permettent de l'établir (la forme des oreilles...) ne se pose pas. L'accusation «fait travailler » un lien entre ceux qui 
étaient présents sur la photo en question. Les photographies étaient utilisées pour élargir le cercle des coupables, pour y inclure de nouveaux " subversifs » et procéder à de nouvelles arrestations. Il va de soi que, compte tenu de cette «méthode", le cercle des coupables peut s'élargir à l'infini. De toute façon, il ne s'agit pas d'identifier un criminel dont on connaît le crime et ainsi mettre fin à l'enquête, mais d'inculper les sujets photographiés parce qu'ils apparaissent sur la photographie à côté du présumé coupable.

\section{L'acte photographique comme émotion partagée} que la source de l'émotion se situe dans l'individu, de voir en elle l'émanation de son intériorité. La place de l'émotion est entre l'intériorité et l'extériorité. Le propre de l'émotion est d'être partagée, en reliant des individus dans un réseau émotionnel. Avec l'affectivité, l'émotion constitue le moteur de l'individuation, elle fait évoluer le préindividuel en direction du transindividuel :

$\mathrm{Si}$, en fait, l'émotion pose à la psychologie des problèmes si difficiles à résoudre, c'est parce qu'elle ne peut être expliquée en fonction de l'être considéré comme totalement individué. Elle manifeste dans l'être individué la rémanence du préindividuel ; elle est ce potentiel réel qui, au sein de l'indéterminé naturel, suscite dans le sujet la relation au sein du collectif qui s'institue; il y a collectif dans la mesure où une émotion se structure... l'émotion est du préindividuel manifesté au sein du sujet, et pouvant être interprété comme intériorité ou extériorité; l'émotion renvoie à l'extériorité et à l'intériorité, parce que l'émotion n'est pas de l'individué ; elle est l'échange, au sein du sujet, entre la charge de nature et les structures stables de l'être individué ; échange entre le préindividuel et l'individué, elle préfigure la découverte du collectif. Elle est une mise en question de l'être en tant qu'individuel, parce qu'elle est pouvoir de susciter une individuation du collectif qui recouvrira et attachera l'être individué ${ }^{16}$.

\section{BIBLIOGRAPHIE}

Bertillon Alphonse, La photographie judiciaire, avec un appendice sur la classification et l'identification anthropométriques, Paris, Gauthier-Villars et fils (Bibliothèque photographique), 1890.

Bourdieu Pierre, Boltanski Luc, Castel Robert, et al., Un art moyen : essai sur les usages sociaux de la photographie, Paris, Minuit, 1965.

Figes Orlando, Les chuchoteurs. Vivre et survivre sous Staline, Pierre-Emmanuel Dauzat (trad.), Emmanuel Carrère (préface), Paris, Deno:̈l, 2009. 
Lukács Georg, Histoire et conscience de classe. Essais de dialectique marxiste, Kostas Axelos et Jacqueline Bois (trad.), Paris, Minuit, 1960.

Lukács Georg, La Pensée de Lénine, Jean-Marie Brohm et Boris Fraenkel (trad.), Paris, DenoëlGonthier (Médiations), 1972.

Phéline Christian, L'image accusatrice, Brax, Association de critique contemporaine en photographie (Les Cahiers de la photographie), 1985.

Riegl Aloïs, Le portrait de groupe hollandais, Aurélie Duthoo et Étienne Jollet (trad.), Étienne Jollet (préface), Paris, Hazan, 2008.

Schmitt Carl, La notion de politique. Théorie du partisan, Marie-Louise Steinhauser (trad.), Paris, Calmann-Lévy (Liberté de l'esprit), 1989.

Simondon Gilbert, L'Individuation à la lumière des notions de forme et d'information, Grenoble, Jérôme Millon (Krisis), 2005.

Traverso Enzo, À feu et à sang. De la guerre civile européenne, 1914-1945, Paris, Stock, 2007.

\section{NOTES}

1. Cette recherche a été réalisée grâce au soutien généreux du New Europe College (Bucarest).

2. Traverso Enzo, À feu et à sang. De la guerre civile européenne, 1914-1945, Paris, Stock, 2007.

3. Schmitt Carl, La notion de politique. Théorie du partisan, Marie-Louise Steinhauser (trad.), Paris, Calmann-Lévy (Liberté de l'esprit), 1989, 329 p., p. 109.

4. Voir Lukács Georg, La Pensée de Lénine, Jean-Marie Brohm et Boris Fraenkel (trad.), Paris, Denoël-Gonthier (Médiations), 1972, 159 p.

5. Lukács Georg, Histoire et conscience de classe. Essais de dialectique marxiste, Kostas Axelos et Jacqueline Bois (trad.), Paris, Minuit, 1960, p. 70.

6. Ibid., p. 87.

7. Simondon Gilbert, L'Individuation à la lumière des notions de forme et d'information, Grenoble, Jérôme Millon (Krisis), 2005, 571 p.

8. Ibid., p. 298.

9. Bourdieu Pierre, Boltanski Luc, Castel Robert, et al., Un art moyen : essai sur les usages sociaux de la photographie, Paris, Minuit, 1965, $368 \mathrm{p}$.

10. Riegl Aloïs, Le portrait de groupe hollandais, Aurélie Duthoo et Étienne Jollet (trad.), Étienne Jollet (préface), Paris, Hazan, 2008, p. 132.

11. Lors d'une rencontre avec Staline, un jeune ouvrier avoua que, bien qu'il fût le fils d'un koulak, il participait avec enthousiasme à la construction socialiste et partageait les valeurs de sa génération. Staline lui répondit par la formule en question.

12. Figes Orlando, Les chuchoteurs. Vivre et survivre sous Staline, Pierre-Emmanuel Dauzat (trad.), Emmanuel Carrère (préface), Paris, DenoËl, 2009, p. 361.

13. Ibid., p. 361.

14. Bertillon Alphonse, La photographie judiciaire, avec un appendice sur la classification et l'identification anthropométriques, Paris, Gauthier-Villars et fils (Bibliothèque photographique), 1890,115 p.

15. Phéline Christian, L'image accusatrice, Brax, Association de critique contemporaine en photographie (Les Cahiers de la photographie), 1985, 169 p.

16. Simondon Gilbert, L'Individuation à la lumière des notions de forme et d'information, p. 314. 


\section{RÉSUMÉS}

Cet article analyse, dans la perspective politique et esthétique, le phénomène de la destruction des visages des « ennemis du peuple » sur les photographies de groupe dans la Russie stalinienne. Il a pour base empirique les photographies découvertes par l'auteur au cours des recherches aux archives de plusieurs villes russes, ces photographies portant différentes traces, telles que les biffages ou les notes manuscrites laissées par les policiers staliniens. L'analyse de ces traces ouvre plusieurs pistes de réflexion, en rendant possible une interrogation conjointe sur la nature de la terreur et la signification politique de la photographie.

INDEX

Mots-clés : photographie, aspect politique, URSS et photographie, xxe siècle et iconoclasme, URSS - totalitarisme et art

\section{AUTEUR}

\section{DENIS SKOPIN}

Maître de conférences, Université d'État de Saint-Pétersbourg 